\title{
On the completeness and Riesz basis property of root subspaces of boundary value problems for first order systems and applications
}

\author{
Anton A. Lunyov and Mark M. Malamud
}

\begin{abstract}
The paper is concerned with the completeness property of root functions of general boundary value problems for $n \times n$ first order systems of ordinary differential equations on a finite interval. In comparison with the recent paper [37] we substantially relax the assumptions on boundary conditions guaranteeing the completeness of root vectors, allowing them to be non-weakly regular and even degenerate. Emphasize that in this case the completeness property substantially depends on the values of a potential matrix at the endpoints of the interval.

It is also shown that the system of root vectors of the general $n \times n$ Dirac type system subject to certain boundary conditions forms a Riesz basis with parentheses. Finally, we apply our results to the dynamic generator of spatially non-homogenous damped Timoshenko beam model.
\end{abstract}

Mathematics Subject Classification (2010). 47E05, 34L10, 35L35.

Keywords. Systems of ordinary differential equations; regular boundary conditions; completeness of root vectors; Riesz basis property; Timoshenko beam model.

\section{Contents}

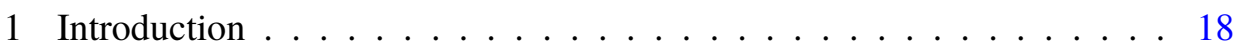

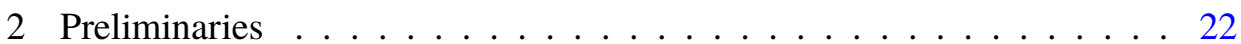

3 Asymptotic behavior of solutions and characteristic determinant . . . 24

4 Explicit completeness results . . . . . . . . . . . . . . 36

5 The Riesz basis property for root functions . . . . . . . . . . . 46

6 Application to the Timoshenko beam model . . . . . . . . . . . 55

References . . . . . . . . . . . . . . . . . . 65 


\section{Introduction}

Spectral theory of non-selfadjoint boundary value problems (BVP) on a finite interval $\mathcal{J}=(a, b)$ for $n$th order ordinary differential equations (ODE)

$$
y^{(n)}+q_{1} y^{(n-2)}+\cdots+q_{n-1} y=\lambda^{n} y, \quad x \in(a, b),
$$

with coefficients $q_{j} \in L^{1}(a, b)$ takes its origin in the papers by Birkhoff $[3,4]$ and Tamarkin [55, 56]. They introduced the concept of regular boundary conditions for ODE and investigated the asymptotic behavior of eigenvalues and eigenfunctions of related BVP. Moreover, they proved that the system of root functions, i.e. eigenfunctions and associated functions, of the regular BVP is complete. Their results are also treated in the classical monographs (see [45, Section 2] and [14, Chapter 19]).

The completeness property of non-regular BVP for $n$th order ODE (1.1) has been studied by M.V. Keldysh [21], A.A. Shkalikov [49], A.G. Kostyuchenko and A.A. Shkalikov [25], G.M. Gubreev [19], A.P. Khromov [23], V.S. Rykhlov [47] and many others (see references in [23]). On the other hand, the Riesz basis property for regular BVP were investigated by N. Dunford [13], V.P. Mikhailov [41], G.M. Kesel'man [22], N. Dunford and J. Schwartz [14, Chapter 19.4], A.A. Shkalikov [50, 51], A. Minkin [42, 43]. Numerous papers are devoted to the completeness and Riesz basis property for the Sturm-Liouville operator (see the recent paper [53] by A. Shkalikov and O. Veliev and the review [33] by A.S. Makin and the references therein). We especially mention the recent achievements for periodic (anti-periodic) Sturm-Liouville operator $-\frac{d^{2}}{d x^{2}}+q(x)$ on $[0, \pi]$. Namely, F. Gesztesy and V.A. Tkachenko $[15,16]$ for $q \in L^{2}[0, \pi]$ and later on P. Djakov and B.S. Mityagin [11] for $q \in W^{-1,2}[0, \pi]$ established by different methods a criterion for the system of root functions to contain a Riesz basis (see Remark 5.12 for detailed discussion).

In this paper we consider first order system of ODE of the form

$$
L y:=L(Q) y:=-i B^{-1} y^{\prime}+Q(x) y=\lambda y, \quad y=\operatorname{col}\left(y_{1}, \ldots, y_{n}\right),
$$

where $B$ is a nonsingular diagonal $n \times n$ matrix with complex entries,

$$
B=\operatorname{diag}\left(b_{1}, b_{2}, \ldots, b_{n}\right) \in \mathbb{C}^{n \times n},
$$

and $Q(\cdot)=:\left(q_{j k}(\cdot)\right)_{j, k=1}^{n} \in L^{1}\left([0,1] ; \mathbb{C}^{n \times n}\right)$ is a potential matrix.

Note that, systems (1.2) form a more general object than ordinary differential equations. Namely, the $n$th order ODE (1.1) can be reduced to the system (1.2) with $b_{j}=\exp (2 \pi i j / n)$ (see [34]). Nevertheless, in general a BVP for ODE (1.1) 
is not reduced to a BVP (1.2)-(1.4) (see below). Systems (1.2) are of significant interest in some theoretical and practical questions. For instance, if $n=2 m$, $B=\operatorname{diag}\left(-I_{m}, I_{m}\right)$ and $Q=\left(\begin{array}{cc}0 & Q_{12} \\ Q_{21} & 0\end{array}\right)$, system (1.2) is equivalent to the Dirac system (see [27, Section VII.1], [38, Section 1.2]). Note also that equation (1.2) is used to integrate the $N$-waves problem arising in nonlinear optics [46, Sec. III.4].

With system (1.2) one associates, in a natural way, the maximal operator $L=$ $L(Q)$ acting in $L^{2}\left([0,1] ; \mathbb{C}^{n}\right)$ on the domain

$$
\operatorname{dom}(L)=\left\{y \in W^{1,1}\left([0,1] ; \mathbb{C}^{n}\right): L y \in L^{2}\left([0,1] ; \mathbb{C}^{n}\right)\right\} .
$$

To obtain a BVP, equation (1.2) is subject to the following boundary conditions

$$
C y(0)+D y(1)=0, \quad C=\left(c_{j k}\right), \quad D=\left(d_{j k}\right) \in \mathbb{C}^{n \times n} .
$$

Denote by

$$
L_{C, D}:=L_{C, D}(Q)
$$

the operator associated in $L^{2}\left([0,1] ; \mathbb{C}^{n}\right)$ with the BVP (1.2)-(1.4). It is defined as the restriction of $L=L(Q)$ to the domain

$$
\operatorname{dom}\left(L_{C, D}\right)=\{y \in \operatorname{dom}(L): C y(0)+D y(1)=0\} .
$$

Moreover, in what follows we always impose the maximality condition

$$
\operatorname{rank}\left(\begin{array}{ll}
C & D
\end{array}\right)=n,
$$

which is equivalent to $\operatorname{ker}\left(C C^{*}+D D^{*}\right)=\{0\}$.

To the best of our knowledge, the spectral problem (1.2)-(1.4) has first been investigated by G.D. Birkhoff and R.E. Langer [5]. Namely, they have extended some previous results of Birkhoff and Tamarkin on non-selfadjoint boundary value problem for ODE (1.1) to the case of BVP (1.2)-(1.4). More precisely, they introduced the concepts of regular and strictly regular boundary conditions (1.4) and investigated the asymptotic behavior of eigenvalues and eigenfunctions of the corresponding operator $L_{C, D}$. Moreover, they proved a pointwise convergence result on spectral decompositions of the operator $L_{C, D}$ corresponding to the BVP (1.2)-(1.4) with regular boundary conditions.

The problem of the completeness of the system of root functions of general $B V P$ (1.2)-(1.4) has first been investigated in the recent papers [36, 37] by one of the authors and L.L. Oridoroga. In these papers the concept of weakly regular boundary conditions for the system (1.2) was introduced and the completeness of root vectors for this class of BVP was proved. During the last decade there appeared numerous papers devoted mainly to the Riesz basis property for 
$2 \times 2$ Dirac system subject to the regular or strictly regular boundary conditions (see $[58,20,2,6,7,9,10,11,12,29,30,48]$ ).

Let us recall the definition of regular (see [5, p. 89]) and weakly regular (see $[36,37])$ boundary conditions. To this end we need the following construction. Let $A=\operatorname{diag}\left(a_{1}, \ldots, a_{n}\right)$ be a diagonal matrix with entries $a_{k}$ (not necessarily distinct) that are not lying on the imaginary axis, $\operatorname{Re} a_{k} \neq 0$. Starting from arbitrary matrices $C, D \in \mathbb{C}^{n \times n}$, we define the auxiliary $n \times n$ matrix $T_{A}(C, D)$ as follows:

- if $\operatorname{Re} a_{k}<0$, then the $k$ th column in the matrix $T_{A}(C, D)$ coincides with the $k$ th column of the matrix $C$;

- if $\operatorname{Re} a_{k}>0$, then the $k$ th column in the matrix $T_{A}(C, D)$ coincides with the $k$ th column of the matrix $D$.

Now consider the lines

$$
l_{j}:=\left\{\lambda \in \mathbb{C}: \operatorname{Re}\left(i b_{j} \lambda\right)=0\right\}, \quad j \in\{1, \ldots, n\},
$$

of the complex plane. They divide the complex plane into $m=2 r \leq 2 n$ sectors. Denote these sectors by $\sigma_{1}, \sigma_{2}, \ldots \sigma_{m}$. Let $z_{j}$ lie in the interior of $\sigma_{j}, j \in$ $\{1, \ldots, m\}$. The boundary conditions (1.4) are called regular whenever

$$
\operatorname{det} T_{i z_{j} B}(C, D) \neq 0, \quad j \in\{1, \ldots, m\} .
$$

We call $z \in \mathbb{C}$ admissible if $\operatorname{Re}\left(i b_{j} z\right) \neq 0$ for $j \in\{1, \ldots, n\}$. Since $T_{i z_{j} B}(C, D)$ does not depend on a particular choice of the point $z_{j} \in \sigma_{j}$, the boundary conditions (1.4) are regular if and only if $\operatorname{det} T_{i z B}(C, D) \neq 0$ for each admissible $z$.

Definition 1.1. ([37]) The boundary conditions (1.4) are called weakly B-regular (or, simply, weakly regular) if there exist three admissible complex numbers $z_{1}, z_{2}$, $z_{3}$ satisfying the following conditions:

(a) the origin is an interior point of the triangle $\triangle_{z_{1} z_{2} z_{3}}$;

(b) $\operatorname{det} T_{i z_{j} B}(C, D) \neq 0$ for $j \in\{1,2,3\}$.

In the case of Dirac type system $\left(B=B^{*}\right)$ the weak regularity of boundary conditions (1.4) is equivalent to their regularity (1.7) and turns into

$$
\operatorname{det} T_{ \pm}:=\operatorname{det} T_{ \pm B}(C, D) \neq 0 .
$$

Therefore, by [37, Theorem 1.2], this condition implies the completeness and minimality in $L^{2}\left([0,1] ; \mathbb{C}^{n}\right)$ of the root functions of BVP (1.2)-(1.4). In special cases 
this statement has earlier been obtained by V. A. Marchenko [38, §1.3] $(2 \times 2$ Dirac system) and V. P. Ginzburg [17] ( $\left.B=I_{n}, Q=0\right)$.

Our first main result (Theorem 4.1) states the completeness property for the general BVP (1.2)-(1.4) with non-weakly regular boundary conditions. It substantially generalizes the corresponding results from [37] and [1]. Emphasize that in the case of non-weakly regular boundary conditions the completeness property substantially depends on the values $Q(0)$ and $Q(1)$. The latter means that Theorem 4.1 cannot be treated as a perturbation theory result: the operator $L_{C, D}(Q)$ satisfying the conditions of this theorem is complete while the system of root vectors of the unperturbed operator $L_{C, D}(0)$ may have infinite defect in $L^{2}\left([0,1] ; \mathbb{C}^{n \times n}\right)$. We demonstrate this fact by the corresponding examples (cf. Corollary 4.7).

Our second main achievement is the Riesz basis property for general $n \times n$ Dirac type system with $Q \in L^{\infty}\left([0,1] ; \mathbb{C}^{n \times n}\right)$ subject to certain boundary conditions. These conditions form rather broad class that covers, in particular, periodic, antiperiodic, and regular splitting (not necessarily selfadjoint) boundary conditions for $2 n \times 2 n$ Dirac system $\left(B=\operatorname{diag}\left(-I_{n}, I_{n}\right)\right.$ ) (see Theorem 5.6 and Proposition 5.9 for the precise statements). Emphasize that to the best of our knowledge even for $2 n \times 2 n$ Dirac systems with $n>1$ the results on the Riesz basis property are obtained here for the first time.

In this connection we mention the series of recent papers by P. Djakov and B.S. Mityagin [7, 9, 10, 11, 12]. In [7] the authors proved that the system of root functions for $2 \times 2$ Dirac system with $Q \in L^{2}\left([0,1] ; \mathbb{C}^{2 \times 2}\right)$ subject to the regular boundary conditions forms a Riesz basis with parentheses while this system forms ordinary Riesz basis provided that the boundary conditions are strictly regular. Moreover, in [9, Theorem 13], [11, Theorem 19] and [12] it is established a criterion for the system of root functions to contain a Riesz basis for periodic (resp., antiperiodic) $2 \times 2$ Dirac operator in terms of the Fourier coefficients of $Q$ as well as in terms of periodic (resp., antiperiodic) and Dirichlet spectra.

Finally, we apply our main abstract results with $B=B^{*} \in \mathbb{C}^{4 \times 4}$ to the Timoshenko beam model investigated under the different restrictions in numerous papers (see [57, 24, 54, 61, 60, 59] and the references therein). We show in Proposition 6.1 that the dynamic generator of this model is similar to the special $4 \times 4$ Dirac type operator. It allows us to derive completeness property in both regular and non-regular cases. Moreover, in the regular case we obtain also the Riesz basis property with parentheses.

The paper is organized as follows. In Section 2 we obtain the general result on completeness that generalizes [37, Theorem 1.2]. In Section 3 we obtain refined asymptotic formulas for solutions of system (1.2) and the characteristic determi- 
nant $\Delta(\cdot)$ of the problem (1.2)-(1.4), provided that the potential matrix $Q(\cdot)$ is continuous at the endpoints 0 and 1.

In Section 4 we prove our main result on completeness, Theorem 4.1. We illustrate this result in $2 \times 2$ case by deriving completeness and minimality in $L^{2}\left([0, \pi] ; \mathbb{C}^{2}\right)$ of the system $\left\{\operatorname{col}\left(e^{a n x} \sin n x, n e^{(a-i) n x}\right)\right\}_{n \in \mathbb{Z} \backslash\{0\}}$. We also obtain some necessary conditions on completeness for general BVP (1.2)-(1.4) generalizing [37, Proposiiton 5.12] and coinciding with it in the case of $2 \times 2$ Dirac system.

In Section 5 we prove the mentioned above results on the Riesz basis property with parentheses for BVP (1.2)-(1.4) with a bounded potential matrix. In Section 6 we prove mentioned above results on the completeness and Riesz basis property with parentheses for the dynamic generator of spatially non-homogenous Timoshenko beam model with both boundary and locally distributed damping.

The main results of the paper were published as a preprint [31] and partially announced in [28].

Notation. $\langle\cdot, \cdot\rangle$ denotes the inner product in $\mathbb{C}^{n} ; \mathbb{C}^{n \times n}$ denotes the set of $n \times n$ matrices with complex entries. $I_{n}\left(\in \mathbb{C}^{n \times n}\right)$ denotes the identity matrix; $\operatorname{GL}(n, \mathbb{C})$ denotes the set of nonsingular matrices from $\mathbb{C}^{n \times n} ; T$ is a closed operator in a Hilbert space $\mathfrak{H} ; \sigma(T)$ and $\rho(T)=\mathbb{C} \backslash \sigma(T)$ denote the spectrum and resolvent set of the operator $T$, respectively; $W^{n, p}[a, b]$ is Sobolev space of functions $f$ having $n-1$ absolutely continuous derivatives on $[a, b]$ and satisfying $f^{(n)} \in L^{p}[a, b]$.

\section{Preliminaries}

Let $\beta_{1}, \ldots, \beta_{r}$ be all different values among $b_{1}, \ldots, b_{n}$. Note that the lines

$$
l_{j k}:=\left\{\lambda \in \mathbb{C}: \operatorname{Re}\left(i \beta_{j} \lambda\right)=\operatorname{Re}\left(i \beta_{k} \lambda\right)\right\}, \quad 1 \leqslant j<k \leqslant r,
$$

together with the lines

$$
l_{j}:=\left\{\lambda \in \mathbb{C}: \operatorname{Re}\left(i \beta_{j} \lambda\right)=0\right\}, \quad j \in\{1, \ldots, r\},
$$

separate $v \leqslant r^{2}+r$ open sectors $S_{p}$ with vertexes at the origin, such that for any $p \in\{1, \ldots, v\}$ the numbers $\beta_{1}, \ldots, \beta_{r}$ can be renumbered so that the following inequalities hold:

$$
\operatorname{Re}\left(i \beta_{j_{1}} \lambda\right)<\cdots<\operatorname{Re}\left(i \beta_{j_{\kappa}} \lambda\right)<0<\operatorname{Re}\left(i \beta_{j_{\kappa}+1} \lambda\right)<\cdots<\operatorname{Re}\left(i \beta_{j_{r}} \lambda\right), \quad \lambda \in S_{p}
$$


Here $\kappa=\kappa_{p}$ is the number of negative values among $\operatorname{Re}\left(i \beta_{1} \lambda\right), \ldots, \operatorname{Re}\left(i \beta_{r} \lambda\right)$ in the sector $S_{p}$. We call $z \in \mathbb{C}$ feasible if $z$ does not belong to any of the lines (2.1) and (2.2), that is, $z$ lies strictly inside some sector $S_{p}$. Note that feasible point is more restrictive notion than admissible point.

Clearly, each of the sectors $S_{p}$ is of the form

$$
S_{p}=\left\{z: \varphi_{1 p}<\arg z<\varphi_{2 p}\right\} .
$$

Denote by $S_{p, \varepsilon}$ a sector strictly embedded into the latter, i.e.,

$$
S_{p, \varepsilon}:=\left\{z: \varphi_{1 p}+\varepsilon<\arg z<\varphi_{2 p}-\varepsilon\right\},
$$

where $\varepsilon>0$ is sufficiently small, and

$$
S_{p, \varepsilon, R}:=\left\{z \in S_{p, \varepsilon}:|z|>R\right\} .
$$

Proposition 2.1. [37, Proposition 2.2] Let $\delta_{j k}$ be a Kronecker symbol, let

$$
\begin{gathered}
B=\operatorname{diag}\left(\beta_{1} I_{n_{1}}, \ldots, \beta_{r} I_{n_{r}}\right), \quad n_{1}+\cdots+n_{r}=n, \\
Q=\left(Q_{j k}\right)_{j, k=1}^{r}, \quad Q_{j k} \in L^{1}\left([0,1] ; \mathbb{C}^{n_{j} \times n_{k}}\right), \\
Q_{j j}(\cdot) \equiv 0, \quad j \in\{1, \ldots, r\} .
\end{gathered}
$$

Let also $p \in\{1, \ldots, v\}$ and let $\varepsilon>0$ be sufficiently small. Then for a sufficiently large $R$, equation (1.2) has a fundamental matrix solution

$$
Y(x, \lambda)=\left(\begin{array}{lll}
Y_{1} & \ldots & Y_{n}
\end{array}\right), \quad Y_{k}(x, \lambda)=\operatorname{col}\left(y_{1 k}, \ldots, y_{n k}\right), \quad k \in\{1, \ldots, n\},
$$

which is analytic in $\lambda \in S_{p, \varepsilon, R}$ and satisfies (uniformly in $x \in[0,1]$ )

$$
y_{j k}(x, \lambda)=\left(\delta_{j k}+o(1)\right) e^{i b_{k} \lambda x}, \quad \text { as } \lambda \rightarrow \infty, \lambda \in S_{p, \varepsilon, R}, j, k \in\{1, \ldots, n\} .
$$

In what follows we will systematically use a concept of the similarity of unbounded operators.

Definition 2.2. Let $\mathfrak{H}_{j}$ be a Hilbert space, $A_{j}$ a closed operator in $\mathfrak{H}_{j}$ with domain $\operatorname{dom}\left(A_{j}\right), j \in\{1,2\}$. The operators $A_{1}$ and $A_{2}$ are called similar if there exists a bounded operator $T$ (a similarity transformation operator) from $\mathfrak{H}_{1}$ onto $\mathfrak{H}_{2}$ with bounded inverse, such that $A_{2}=T A_{1} T^{-1}$, i.e.

$$
\operatorname{dom}\left(A_{2}\right)=T \operatorname{dom}\left(A_{1}\right) \quad \text { and } \quad A_{2} f=T A_{1} T^{-1} f, \quad f \in \operatorname{dom}\left(A_{2}\right) .
$$


Note that similar operators $A_{1}$ and $A_{2}\left(A_{2}=T A_{1} T^{-1}\right)$ have the same spectra, algebraic and geometric multiplicities of eigenvalues, while the systems of their root vectors $\left\{e_{k}^{(j)}\right\}, j \in\{1,2\}$, are related by $e_{k}^{(2)}=T e_{k}^{(1)}$. Therefore, they also have the same geometric properties (completeness, minimality, basis property, etc.).

Next we state the completeness result which slightly generalizes [37, Theorem 1.2]. The proof can be found in our preprint [31] and due to the lack of space is omitted.

Let $\Phi(x, \lambda)$ be a fundamental matrix solution of equation (1.2) satisfying

$$
\Phi(0, \lambda)=I_{n}, \quad \lambda \in \mathbb{C}
$$

The characteristic determinant $\Delta(\cdot)$ of the problem $(1.2)-(1.4)$ is given by

$$
\Delta(\lambda):=\operatorname{det}(C+D \Phi(1, \lambda)), \quad \lambda \in \mathbb{C} .
$$

Theorem 2.3. Let $Q(\cdot) \in L^{1}\left([0,1] ; \mathbb{C}^{n \times n}\right)$. Assume that there exist $C, R>0$, $s \in \mathbb{Z}_{+}$and three feasible numbers $z_{1}, z_{2}, z_{3}$ satisfying the following conditions:

(i) the origin is the interior point of the triangle $\Delta_{z_{1} z_{2} z_{3}}$;

(ii) for $k \in\{1,2,3\}$ we have

$$
|\Delta(\lambda)| \geqslant \frac{C e^{\operatorname{Re}\left(i \tau_{k} \lambda\right)}}{|\lambda|^{s}}, \quad \tau_{k}=\sum_{\substack{j=1 \\ \operatorname{Re}\left(i b_{j} z_{k}\right)>0}}^{n} b_{j}, \quad|\lambda|>R, \arg \lambda=\arg z_{k}
$$

Then the system of root functions of the BVP(1.2)-(1.4) (of the operator $L_{C, D}(Q)$ ) is complete and minimal in $L^{2}\left([0,1] ; \mathbb{C}^{n}\right)$.

Remark 2.4. In the case $s=0$ Theorem 2.3 is implicitly contained in [37, Theorem 1.2].

\section{Asymptotic behavior of solutions and characteristic determinant}

Here we refine asymptotic formulas (2.6) assuming that $Q(\cdot)$ is continuous at the endpoints 0 and 1 . These formulas will be applied to investigate asymptotic behavior of the characteristic determinant $\Delta(\cdot)$. We start with the following lemma. 
Lemma 3.1. Let $b \in \mathbb{C} \backslash\{0\}, C>0$ and $S \subset \mathbb{C}$ be a non-bounded subset of $\mathbb{C}$ such that

$$
\operatorname{Re}(b \lambda)<-C|\lambda|, \quad \lambda \in S .
$$

(i) Let $\varphi \in L^{1}[0,1]$ and $\varphi(\cdot)$ is continuous at zero. Then

$$
\int_{0}^{1} e^{b \lambda t} \varphi(t) d t=\frac{\varphi(0)+o(1)}{-b \lambda}, \quad \text { as } \lambda \rightarrow \infty, \lambda \in S .
$$

(ii) Let $\varphi \in L^{1}[0,1]$ and let $\varphi(\cdot)$ be bounded at a neighborhood of zero. Then

$$
\int_{0}^{1}\left|e^{b \lambda t} \varphi(t)\right| d t=O\left(|\lambda|^{-1}\right), \quad \lambda \in S
$$

Proof. Taking into account (3.1) one has

$$
\begin{aligned}
\int_{0}^{1}\left|e^{b t} \varphi(t)\right| d t & \leqslant\left(\int_{0}^{\delta}+\int_{\delta}^{1}\right) e^{-C|\lambda| t}|\varphi(t)| d t \\
& \leqslant \frac{1}{C|\lambda|} \sup _{t \in[0, \delta]}|\varphi(t)|+\|\varphi\|_{1} e^{-C \delta|\lambda|} .
\end{aligned}
$$

This implies (3.3). Further, (3.2) is true for $\varphi(\cdot) \equiv$ const. Therefore, it is sufficient to prove it in the case $\varphi(0)=0$. Estimate (3.4) proves this, taking into account that $\delta$ can be chosen arbitrary small.

Lemma 3.1 allows us to refine the asymptotic formulas (2.6) from Proposition 2.1 when $Q$ is continuous at the endpoints of the segment $[0,1]$.

Proposition 3.2. Assume conditions (2.5a)-(2.5c) and let $p \in\{1, \ldots, v\}$. Assume, in addition, that $Q$ is continuous at the endpoints 0,1 . Then for a sufficiently large $R$ and small $\varepsilon>0$ equation (1.2) has a fundamental matrix solution

$$
Y(x, \lambda)=\left(\begin{array}{lll}
Y_{1} & \ldots & Y_{n}
\end{array}\right), \quad Y_{k}(x, \lambda)=\operatorname{col}\left(y_{1 k}, \ldots, y_{n k}\right), \quad k \in\{1, \ldots, n\},
$$

analytic with respect to $\lambda \in S_{p, \varepsilon, R}$. Moreover, $y_{j k}(x, \lambda), j, k \in\{1, \ldots, n\}$, satisfies (2.6) and has the following asymptotic behavior at the endpoints 0 and 1 as $\lambda \rightarrow \infty, \lambda \in S_{p, \varepsilon, R}$,

$$
y_{j k}(0, \lambda)= \begin{cases}0, & \text { if } \operatorname{Re}\left(i b_{j} \lambda\right)<\operatorname{Re}\left(i b_{k} \lambda\right), \\ \delta_{j k}, & \text { if } b_{j}=b_{k}, \\ \frac{b_{j} q_{j k}(0)+o(1)}{b_{j}-b_{k}} \cdot \frac{1}{\lambda}, & \text { if } \operatorname{Re}\left(i b_{j} \lambda\right)>\operatorname{Re}\left(i b_{k} \lambda\right) ;\end{cases}
$$


and

$$
y_{j k}(1, \lambda)= \begin{cases}\frac{b_{j} q_{j k}(1)+o(1)}{b_{j}-b_{k}} \cdot \frac{e^{i b_{k} \lambda}}{\lambda}, & \text { if } \operatorname{Re}\left(i b_{j} \lambda\right)<\operatorname{Re}\left(i b_{k} \lambda\right), \\ \left(\delta_{j k}+o(1)\right) e^{i b_{k} \lambda}, & \text { if } b_{j}=b_{k}, \\ 0, & \text { if } \operatorname{Re}\left(i b_{j} \lambda\right)>\operatorname{Re}\left(i b_{k} \lambda\right) .\end{cases}
$$

Proof. According to the proof of [37, Proposition 2.2] the matrix solution $Y(x, \lambda)$ of system (1.2) with the asymptotic behavior (2.6) in $S_{p, \varepsilon, R}$ was constructed as the unique solution of the following system of integral equations

$$
y_{j k}(x, \lambda)=\delta_{j k} e^{i b_{k} \lambda x}-i b_{j} \int_{a_{j k}}^{x} e^{-i b_{j} \lambda(t-x)} \sum_{l=1}^{n} q_{j l}(t) y_{l k}(t, \lambda) d t,
$$

where

$$
a_{j k}:= \begin{cases}0, & \text { if } \operatorname{Re}\left(i b_{j} \lambda\right) \leqslant \operatorname{Re}\left(i b_{k} \lambda\right), \lambda \in S_{p, \varepsilon}, \\ 1, & \text { if } \operatorname{Re}\left(i b_{j} \lambda\right)>\operatorname{Re}\left(i b_{k} \lambda\right), \lambda \in S_{p, \varepsilon} .\end{cases}
$$

In particular, $a_{j k}=0$ if $b_{j}=b_{k}$. Let us show that this solution satisfies (3.5), (3.6). It is clear from (3.7) that for $\lambda \in S_{p, \varepsilon, R}$ we have

$$
\begin{array}{ll}
y_{j k}(0, \lambda)=0, & \operatorname{Re}\left(i b_{j} \lambda\right)<\operatorname{Re}\left(i b_{k} \lambda\right), \\
y_{j k}(0, \lambda)=\delta_{j k}, & b_{j}=b_{k}, \\
y_{j k}(1, \lambda)=0, & \operatorname{Re}\left(i b_{j} \lambda\right)>\operatorname{Re}\left(i b_{k} \lambda\right),
\end{array}
$$

while the second relation in (3.6) follows from Proposition 3.2. Thus, we need to prove only the third relation in (3.5) and the first one in (3.6).

At first we rewrite (2.6) in the following form

$$
y_{j k}(x, \lambda)=\left(\delta_{j k}+\rho_{j k}(x, \lambda)\right) e^{i b_{k} \lambda}, \quad j, k \in\{1, \ldots, n\},
$$

where $\rho_{j k}(x, \lambda)=o(1)$, as $\lambda \rightarrow \infty, \lambda \in S_{p, \varepsilon, R}$, uniformly in $x \in[0,1]$. Now inserting expression (3.9) for $y_{j k}(x, \lambda)$ into (3.7) we obtain

$$
\begin{aligned}
& y_{j k}(x, \lambda) \\
& \quad=\left(\delta_{j k}-i b_{j} \int_{a_{j k}}^{x} e^{i\left(b_{k}-b_{j}\right) \lambda(t-x)}\left(q_{j k}(t)+\sum_{l=1}^{n} q_{j l}(t) \rho_{l k}(t, \lambda)\right) d t\right) e^{i b_{k} \lambda x} .
\end{aligned}
$$

Let $\operatorname{Re}\left(i b_{j} \lambda\right)>\operatorname{Re}\left(i b_{k} \lambda\right)$. Setting $x=0$ in (3.10) one gets

$$
y_{j k}(0, \lambda)=i b_{j} \int_{0}^{1} e^{i\left(b_{k}-b_{j}\right) \lambda t} q_{j k}(t) d t+i b_{j} \int_{0}^{1} e^{i\left(b_{k}-b_{j}\right) \lambda t} \sum_{l=1}^{n} q_{j l}(t) \rho_{l k}(t, \lambda) d t .
$$


Clearly,

$$
\operatorname{Re}\left(i\left(b_{k}-b_{j}\right) \lambda\right)<-C|\lambda|, \quad \lambda \in S_{p, \varepsilon, R},
$$

for some $C>0$. Hence, applying Lemma 3.1(i) with

$$
S=S_{p, \varepsilon, R}, \quad b=i\left(b_{k}-b_{j}\right), \quad \varphi(\cdot)=i b_{j} q_{j k}(\cdot),
$$

and taking into account the continuity of $q_{j k}(\cdot)$ at zero, we derive from (3.2)

$$
i b_{j} \int_{0}^{1} e^{i\left(b_{k}-b_{j}\right) \lambda t} q_{j k}(t) d t=\frac{b_{j} q_{j k}(0)+o(1)}{\left(b_{j}-b_{k}\right) \lambda}, \quad \text { as } \lambda \rightarrow \infty, \lambda \in S_{p, \varepsilon, R} .
$$

Further, since $q_{j l}(\cdot), l \in\{1, \ldots, n\}$, is bounded at a neighborhood of zero and

$$
\sup _{t \in[0,1]}\left|\rho_{l k}(t, \lambda)\right|=o(1) \quad \text { as } \lambda \rightarrow \infty, \lambda \in S_{p, \varepsilon, R},
$$

Lemma 3.1(ii) implies

$$
\begin{aligned}
\int_{0}^{1} e^{i\left(b_{k}-b_{j}\right) \lambda t} \sum_{l=1}^{n} q_{j l}(t) \rho_{l k}(t, \lambda) d t & =\sum_{l=1}^{n} o\left(\int_{0}^{1}\left|e^{i\left(b_{k}-b_{j}\right) \lambda t} q_{j l}(t)\right| d t\right) \\
& =o\left(\lambda^{-1}\right),
\end{aligned}
$$

as $\lambda \rightarrow \infty, \lambda \in S_{p, \varepsilon, R}$. This together with (3.11) and (3.12) yields the first relation in (3.5). Next, let $\operatorname{Re}\left(i b_{j} \lambda\right)<\operatorname{Re}\left(i b_{k} \lambda\right)$. Then using (3.8) we obtain from (3.10)

$$
\begin{aligned}
& y_{j k}(1, \lambda) \\
& \quad=-i b_{j} e^{i b_{k} \lambda} \int_{0}^{1} e^{i\left(b_{j}-b_{k}\right) \lambda s}\left(q_{j k}(1-s)+\sum_{l=1}^{n} q_{j l}(1-s) \rho_{l k}(1-s, \lambda)\right) d s .
\end{aligned}
$$

Using the inequality $\operatorname{Re}\left(i\left(b_{j}-b_{k}\right) \lambda\right)<-C|\lambda|, \lambda \in S_{p, \varepsilon, R}$, and continuity of $q_{j l}(\cdot), l \in\{1, \ldots, n\}$, at the point 1 , and follow the above reasoning we arrive at the third relation in (3.6).

Remark 3.3. Fix $j, k \in\{1, \ldots, n\}$. As it is clear from the proof of Proposition 3.2, the individual function $y_{j, k}(x, \lambda)$ satisfies the third relation in (3.5) whenever $q_{j k}(\cdot)$ is continuous at zero and $q_{j l}(\cdot)$ is bounded at zero for $l \in\{1, \ldots, n\}$. Otherwise it satisfies only the weaker relation $y_{j k}(0, \lambda)=o(1)$ as $\lambda \rightarrow \infty, \lambda \in S_{p, \varepsilon}$. Moreover, if $q_{j l}(\cdot), l \in\{1, \ldots, n\}$, is just bounded at zero then, by Lemma 3.1(ii), $y_{j k}(0, \lambda)=O\left(\lambda^{-1}\right), \lambda \in S_{p, \varepsilon, R}$. Similar statements are true for $y_{j k}(1, \lambda)$. This allows us to weaken assumptions on $Q(\cdot)$ in further considerations. 
Next we investigate the asymptotic behavior of the characteristic determinant $\Delta(\cdot)$. Emphasize that in contrast to the considerations of the previous paper [37], in the following proposition we ignore block matrix structure $(2.5 \mathrm{a})-(2.5 \mathrm{~b})$, i.e. do not assume that equal $b_{j}$ th are grouped into the blocks. It is motivated by the application to the Timoshenko beam model in Section 6 where such situation naturally occurs for matrix (6.12a) with equal eigenvalues $b_{1}=b_{2}$.

Proposition 3.4. Let $B$ be defined by (1.3), $Q(\cdot) \in L^{1}\left([0,1] ; \mathbb{C}^{n \times n}\right)$ and let $q_{j k}$ be continuous at points 0 and 1 if $b_{j} \neq b_{k}$. Let, as above, $\Delta(\cdot)$ be the characteristic determinant (2.9) of the problem (1.2)-(1.4). Finally, let $p \in\{1, \ldots, v\}$. Then for sufficiently small $\varepsilon>0$ the characteristic determinant $\Delta(\cdot)$ admits the following asymptotic expansion

$$
\Delta(\lambda)=\gamma_{p} \cdot\left(\omega_{0}\left(z_{p}\right) \cdot(1+o(1))+\frac{\omega_{1}\left(z_{p}\right)+o(1)}{\lambda}\right) e^{i \tau_{p} \lambda}, \quad \text { as } \lambda \rightarrow \infty, \lambda \in S_{p, \varepsilon} .
$$

Here $z_{p}$ is a fixed point in $S_{p, \varepsilon}$,

$$
\begin{aligned}
\gamma_{p} & :=\exp \left(\sum_{\operatorname{Re}\left(i b_{j} z_{p}\right)>0} i b_{j} \int_{0}^{1} q_{j j}(t) d t\right), \\
\tau_{p} & :=\sum_{\operatorname{Re}\left(i b_{j} z_{p}\right)>0} b_{j}, \\
\omega_{0}\left(z_{p}\right) & :=\operatorname{det} T_{i z_{p} B}(C, D), \\
\omega_{1}\left(z_{p}\right) & :=\sum_{\substack{\operatorname{Re}\left(i b_{j} z_{p}\right)<0 \\
\operatorname{Re}\left(i b_{k} z_{p}\right)>0}} \frac{\operatorname{det} T_{i z_{p} B}^{c_{j} \rightarrow c_{k}} b_{k} q_{k j}(0)-\operatorname{det} T_{i z_{p} B}^{d_{k} \rightarrow d_{j}} b_{j} q_{j k}(1)}{b_{k}-b_{j}},
\end{aligned}
$$

and the matrix $T_{i z_{p} B}^{c_{j} \rightarrow c_{k}}\left(T_{i z_{p} B}^{d_{j} \rightarrow d_{k}}\right)$ is obtained from $T_{i z_{p} B}(C, D)$ by replacing its $j$ th column by the kth column of the matrix $C$ (resp. D).

Remark 3.5. Denote by $c_{j}\left(d_{j}\right)$ the $j$ th column of the matrix $C$ (resp. $D$ ). Note that if $\operatorname{Re}\left(i b_{j} \lambda\right)<0$, the $j$ th column of $T_{i z_{p} B}(C, D)$ coincides with $c_{j}$. Therefore, the superscript $c_{j} \rightarrow c_{k}$ in the notation of the matrix $T_{i z_{p} B}^{c_{j} \rightarrow c_{k}}$ means just replacement $c_{j}$ by $c_{k}$ in $T_{i z_{p} B}$. The notation $T_{i z_{p} B}^{d_{k} \rightarrow d_{j}}$ is justified similarly. 
Proof of Proposition 3.4. By renumbering $y_{1}, \ldots, y_{n}$ we can assume that the matrix $B$ satisfies (2.5a) and hence $Q$ has representation (2.5b). Let

$$
Q_{1}(x):=\operatorname{diag}\left(Q_{11}(x), \ldots, Q_{r r}(x)\right)
$$

and let $W(\cdot)$ be the solution to the Cauchy problem

$$
i B^{-1} W^{\prime}=Q_{1}(x) W, \quad W(0)=I_{n} .
$$

Due to the block structure of the matrices $B$ and $Q_{1}$ one easily derives

$$
W(x)=\operatorname{diag}\left(W_{11}(x), \ldots, W_{r r}(x)\right), \quad W_{j j}(x) \in \mathrm{GL}\left(n_{j}, \mathbb{C}\right), \quad x \in[0,1] .
$$

Denoting by $W: y \rightarrow W(x) y$ the gauge transform and letting

$$
\widetilde{D}:=D W(1) \quad \text { and } \quad \tilde{Q}(x)=W^{-1}(x)\left(Q(x)-Q_{1}(x)\right) W(x)=:\left(\tilde{q}_{j k}(x)\right)_{j, k=1}^{n}
$$

we get

$$
L_{C, \tilde{D}}(\tilde{Q})=W^{-1} L_{C, D}(Q) W
$$

i.e. $L_{C, D}(Q)$ and $L_{C, \tilde{D}}(\tilde{Q})$ are similar. Clearly,

$$
\widetilde{\Phi}=: W^{-1} \Phi
$$

is a fundamental solution of equation (1.2) with $\widetilde{Q}$ in place of $Q$ and the corresponding characteristic determinant $\widetilde{\Delta}(\cdot)$ (see (2.9)) is

$$
\widetilde{\Delta}(\lambda):=\operatorname{det}(C+\widetilde{D} \widetilde{\Phi}(1, \lambda))=\operatorname{det}\left(C+D W(1) W^{-1}(1) \Phi(1, \lambda)\right)=\Delta(\lambda) .
$$

Further, $Q-Q_{1}$ is continuous at the endpoints 0 and 1 . Since both $W(\cdot)$ and $W^{-1}(\cdot)$ are continuous on $[0,1], \widetilde{Q}$ is continuous at the endpoints 0 and 1 too. According to (3.21) $\widetilde{Q}$ satisfies (2.5c) and, by Proposition 3.2, there exists a fundamental matrix solution $\tilde{Y}(\cdot, \lambda)$ of system $(1.2)$ with $\widetilde{Q}$ in place of $Q$, that satisfies asymptotic relations (3.5) and (3.6) with $\tilde{q}_{j k}(\cdot)$ in place of $q_{j k}(\cdot)$. The fundamental matrices $\tilde{Y}(\cdot, \lambda)$ and $\widetilde{\Phi}(\cdot, \lambda)$ are related by

$$
\tilde{Y}(x, \lambda)=\widetilde{\Phi}(x, \lambda) P(\lambda), \quad x \in[0,1], \lambda \in S_{p, \varepsilon, R},
$$

where $P(\lambda)=:\left(p_{k j}(\lambda)\right)_{k, j=1}^{n}$ is an analytical invertible matrix function in $S_{p, \varepsilon, R}$. Hence $\tilde{Y}(0, \lambda)=P(\lambda)$ and due to (2.6) and (3.22) (cf. [37, formula (3.31)]),

$$
\Delta_{\tilde{Y}}(\lambda):=\operatorname{det}(C \tilde{Y}(0, \lambda)+\tilde{D} \tilde{Y}(1, \lambda))=\tilde{\Delta}(\lambda) \operatorname{det}(\tilde{Y}(0, \lambda))=(1+o(1)) \Delta(\lambda),
$$


as $\lambda \rightarrow \infty, \lambda \in S_{p, \varepsilon}$. Thus, it suffices to prove (3.14) with $\Delta_{\tilde{Y}}(\cdot)$ instead of $\Delta(\cdot)$. Since $W(0)=I_{n}$, one has $\widetilde{Q}(0)=Q(0)-Q_{1}(0)$ and hence

$$
\tilde{Y}(0, \lambda)=Y_{0}:=Y_{0}(\lambda):=\left(y_{j k}^{[0]}(\lambda)\right)_{j, k=1}^{n},
$$

where $y_{j k}^{[0]}(\lambda)$ is given by (3.5). Let us simplify $\tilde{Y}(1, \lambda)$. To this end let

$$
\begin{aligned}
& \widetilde{Q}(x)=\left(\tilde{Q}_{j k}(x)\right)_{j, k=1}^{r}, \quad \widetilde{Q}_{j k}(x) \in \mathbb{C}^{n_{j} \times n_{k}}, \\
& \tilde{Y}(x, \lambda)=\left(\tilde{Y}_{j k}(x, \lambda)\right)_{j, k=1}^{r}, \quad \tilde{Y}_{j k}(x, \lambda) \in \mathbb{C}^{n_{j} \times n_{k}},
\end{aligned}
$$

be the block-representations of matrices $\widetilde{Q}(x)$ and $\tilde{Y}(x, \lambda)$ with respect to the orthogonal decomposition $\mathbb{C}^{n}=\mathbb{C}^{n_{1}} \oplus \cdots \oplus \mathbb{C}^{n_{r}}$. It follows from (3.20)-(3.21) that

$$
\widetilde{Q}_{j k}(1)=W_{j j}^{-1}(1) Q_{j k}(1) W_{k k}(1), \quad j \neq k .
$$

Further, note that due to $(2.5 \mathrm{a})-(2.5 \mathrm{~b})$ formula $(3.6)$ for $\tilde{Y}(1, \lambda)$ takes the form

$$
\tilde{Y}_{j k}(1, \lambda)= \begin{cases}\frac{\beta_{j} \widetilde{Q}_{j k}(1)+o(1)}{\beta_{j}-\beta_{k}} \cdot \frac{e^{i \beta_{k} \lambda}}{\lambda}, & \text { if } \operatorname{Re}\left(i \beta_{j} \lambda\right)<\operatorname{Re}\left(i \beta_{k} \lambda\right), \\ \left(I_{n_{k}}+o(1)\right) e^{i \beta_{k} \lambda}, & \text { if } j=k, \\ 0, & \text { if } \operatorname{Re}\left(i \beta_{j} \lambda\right)>\operatorname{Re}\left(i \beta_{k} \lambda\right) .\end{cases}
$$

In view of (2.5a)-(2.5b) and (3.26)-(3.27) we have

$$
\tilde{Y}(1, \lambda)=W^{-1}(1) Y_{1} W(1), \quad Y_{1}:=Y_{1}(\lambda)=\left(y_{j k}^{[1]}(\lambda)\right)_{j, k=1}^{n},
$$

where $y_{j k}^{[1]}(\lambda)$ is given by (3.6). Combining (3.21), (3.24), (3.25) and (3.28) yields

$$
\Delta_{\tilde{Y}}(\lambda)=\operatorname{det}\left(C Y_{0}(\lambda)+D Y_{1}(\lambda) W(1)\right)=\operatorname{det}(J \cdot V),
$$

where $V:=V(\lambda):=\left(\begin{array}{l}Y_{0} \\ V_{1}\end{array}\right), V_{1}:=Y_{1} W(1)$, and $J:=\left(\begin{array}{ll}C & D\end{array}\right)$. By the Cauchy-Binet formula

$$
\Delta_{\tilde{Y}}(\lambda)=\sum_{1 \leqslant k_{1}<\ldots<k_{n} \leqslant 2 n} J\left(\begin{array}{cccc}
1 & 2 & \ldots & n \\
k_{1} & k_{2} & \ldots & k_{n}
\end{array}\right) \cdot V\left(\begin{array}{cccc}
k_{1} & k_{2} & \ldots & k_{n} \\
1 & 2 & \ldots & n
\end{array}\right) .
$$

Here $A\left(\begin{array}{llll}j_{1} & j_{2} & \ldots & j_{p} \\ k_{1} & k_{2} & \ldots & k_{p}\end{array}\right)$ denotes the minor of $n \times n^{\prime}$ matrix $A=\left(a_{j k}\right)$ composed of its entries located in the rows with indices $j_{1}, \ldots, j_{p} \in\{1, \ldots, n\}$ and columns with indices $k_{1}, \ldots, k_{p} \in\left\{1, \ldots, n^{\prime}\right\}$. 
Fix a set $\left\{k_{1}, k_{2}, \ldots, k_{n}\right\}$ such that $1 \leqslant k_{1}<\cdots<k_{n} \leqslant 2 n$ and denote by $m$ the number of entries of the set that do not exceed $n$, i.e., $1 \leqslant k_{1}<\cdots<k_{m} \leqslant$ $n<k_{m+1}<\cdots<k_{n}$. Applying Laplace theorem to expand the second factor in (3.30) with respect to the first $m$ rows, one gets

$$
\begin{aligned}
V & \left(\begin{array}{cccc}
k_{1} & k_{2} & \ldots & k_{n} \\
1 & 2 & \ldots & n
\end{array}\right) \\
= & \sum_{\substack{\left.1 \leqslant j_{1}<\ldots j_{m} \leqslant n \\
1 \leqslant j_{m}+1<\ldots<j n<n \\
j_{1}, \ldots, j_{n}\right\}=\{1, \ldots, n\}}}(-1)^{(1+\cdots+m)+\left(j_{1}+\cdots+j_{m}\right)} \\
& \times Y_{0}\left(\begin{array}{ccc}
k_{1} & \ldots & k_{m} \\
j_{1} & \ldots & j_{m}
\end{array}\right) \cdot V_{1}\left(\begin{array}{ccc}
k_{m+1}-n & \ldots & k_{n}-n \\
j_{m+1} & \ldots & j_{n}
\end{array}\right) .
\end{aligned}
$$

It follows from (3.5) and (3.6) that

$$
y_{j k}^{[0]}(\lambda)=O(1), \quad y_{j k}^{[1]}(\lambda)=O(1) \cdot e^{i b_{k} \lambda}, \quad \lambda \in S_{p, \varepsilon, R}, j, k \in\{1, \ldots, n\} .
$$

Setting $\left(v_{j k}(\lambda)\right)_{j, k=1}^{n}:=V_{1}(\lambda)=Y_{1}(\lambda) W(1)$ we obtain from (3.32) and the blockdiagonal structure of the matrices $B$ and $W(1)$ that

$$
v_{j k}(\lambda)=O(1) \cdot e^{i b_{k} \lambda}, \quad \lambda \in S_{p, \varepsilon, R}, j, k \in\{1, \ldots, n\} .
$$

It follows from (3.25), (3.28), (3.32), (3.33) that for $\lambda \in S_{p, \varepsilon, R}$

$$
Y_{0}\left(\begin{array}{ccc}
k_{1} & \ldots & k_{m} \\
j_{1} & \ldots & j_{m}
\end{array}\right)=O(1)
$$

and

$$
V_{1}\left(\begin{array}{ccc}
k_{m+1}-n & \ldots & k_{n}-n \\
j_{m+1} & \ldots & j_{n}
\end{array}\right)=O(1) \cdot e^{i\left(b_{j_{m+1}}+\cdots+b_{j_{n}}\right) \lambda} .
$$

Let $\kappa$ be a number of negative values among $\operatorname{Re}\left(i b_{1} \lambda\right), \ldots, \operatorname{Re}\left(i b_{n} \lambda\right), \lambda \in S_{p, \varepsilon}$. For definiteness we assume that

$$
\operatorname{Re}\left(i b_{j} \lambda\right)<0, \quad j \in\{1, \ldots, \kappa\}
$$

and

$$
\operatorname{Re}\left(i b_{j} \lambda\right)>0, \quad j \in\{\kappa+1, \ldots, n\}
$$


It is clear from (3.36) that for $\left\{j_{m+1}, \ldots, j_{n}\right\} \neq\{\kappa+1, \ldots, n\}$ the following inequality holds

$$
\begin{aligned}
\operatorname{Re}\left(i b_{j_{m+1}} \lambda\right)+\cdots+\operatorname{Re}\left(i b_{j_{n}} \lambda\right) & <\operatorname{Re}\left(i b_{\kappa+1} \lambda\right)+\cdots+\operatorname{Re}\left(i b_{n} \lambda\right) \\
& =\operatorname{Re}\left(i \tau_{p} \lambda\right), \quad \lambda \in S_{p, \varepsilon}
\end{aligned}
$$

where $\tau_{p}$ is given by (3.16). Combining this estimate with (3.34) and (3.35) yields that for $\left\{j_{m+1}, \ldots, j_{n}\right\} \neq\{\kappa+1, \ldots, n\}$ and each $h \in \mathbb{N}$,

$Y_{0}\left(\begin{array}{ccc}k_{1} & \ldots & k_{m} \\ j_{1} & \ldots & j_{m}\end{array}\right) \cdot V_{1}\left(\begin{array}{ccc}k_{m+1}-n & \ldots & k_{n}-n \\ j_{m+1} & \ldots & j_{n}\end{array}\right)=O\left(\frac{1}{\lambda^{h}}\right) \cdot e^{i \tau_{p} \lambda}, \quad \lambda \in S_{p, \varepsilon, R}$

Inserting (3.37) into (3.31) we obtain for $\lambda \in S_{p, \varepsilon, R}$ and each $h \in \mathbb{N}$ that

$$
V\left(\begin{array}{ccc}
k_{1} & \ldots & k_{n} \\
1 & \ldots & n
\end{array}\right)=O\left(\frac{1}{\lambda^{h}}\right) \cdot e^{i \tau_{p} \lambda}, \quad m \neq \kappa
$$

and

$$
\begin{gathered}
V\left(\begin{array}{ccc}
k_{1} & \ldots & k_{n} \\
1 & \ldots & n
\end{array}\right)=Y_{0}\left(\begin{array}{ccc}
k_{1} & \ldots & k_{\kappa} \\
1 & \ldots & \kappa
\end{array}\right) \cdot V_{1}\left(\begin{array}{ccc}
k_{\kappa+1}-n & \ldots & k_{n}-n \\
\kappa+1 & \ldots & n
\end{array}\right) \\
+O\left(\frac{1}{\lambda^{h}}\right) \cdot e^{i \tau_{p} \lambda}, \quad m=\kappa,
\end{gathered}
$$

Due to the block-diagonal structure of $W(1)$ one has

$$
V_{1}\left(\begin{array}{ccc}
k_{\kappa+1} & \ldots & k_{n} \\
\kappa+1 & \ldots & n
\end{array}\right)=Y_{1}\left(\begin{array}{ccc}
k_{\kappa+1} & \ldots & k_{n} \\
\kappa+1 & \ldots & n
\end{array}\right) \gamma(\lambda)
$$

where

$$
\gamma(\lambda):=\prod_{\substack{j=1 \\ \operatorname{Re}\left(i \beta_{j} \lambda\right)>0}}^{r} \operatorname{det} W_{j j}(1)
$$

Applying the Liouville theorem to system (3.19) and using the definition of the sector $S_{p, \varepsilon}$ yields $\gamma(\lambda)=\gamma_{p}, \lambda \in S_{p, \varepsilon}$, where $\gamma_{p}$ is given by (3.15). Now it follows from (3.30), (3.38), (3.39) and (3.40) that for $\lambda \in S_{p, \varepsilon, R}$ 


$$
\begin{aligned}
\Delta_{\tilde{Y}}(\lambda)=\gamma_{p} & \sum_{\substack{1 \leqslant k_{1}<\ldots<k_{\kappa} \leqslant n \\
1 \leqslant k_{\kappa+1}<\ldots<k n \leqslant n}} J\left(\begin{array}{cccccc}
1 & \ldots & \kappa & \kappa+1 & \ldots & n \\
k_{1} & \ldots & k_{\kappa} & n+k_{\kappa+1} & \ldots & n+k_{n}
\end{array}\right) \\
& \times Y_{0}\left(\begin{array}{ccc}
k_{1} & \ldots & k_{\kappa} \\
1 & \ldots & \kappa
\end{array}\right) \cdot Y_{1}\left(\begin{array}{ccc}
k_{\kappa+1} & \ldots & k_{n} \\
\kappa+1 & \ldots & n
\end{array}\right) \\
+ & O\left(\frac{1}{\lambda^{h}}\right) \cdot e^{i \tau_{p} \lambda}, \quad h \in \mathbb{N} .
\end{aligned}
$$

Let $\left(k_{1}, \ldots, k_{\kappa}\right) \in \mathbb{N}^{\kappa}$ be a sequence satisfying $1 \leqslant k_{1}<\cdots<k_{\kappa} \leqslant n$ and let $\left(l_{1}, \ldots, l_{\kappa}\right)$ be its permutation. It is easily seen that

$$
\begin{aligned}
& J\left(\begin{array}{cccccc}
1 & \ldots & \kappa & \kappa+1 & \ldots & n \\
k_{1} & \ldots & k_{\kappa} & n+k_{\kappa+1} & \ldots & n+k_{n}
\end{array}\right) \cdot Y_{0}\left(\begin{array}{ccc}
k_{1} & \ldots & k_{\kappa} \\
1 & \ldots & \kappa
\end{array}\right) \\
& \quad=J\left(\begin{array}{cccccc}
1 & \ldots & \kappa & \kappa+1 & \ldots & n \\
l_{1} & \ldots & l_{\kappa} & n+k_{\kappa+1} & \ldots & n+k_{n}
\end{array}\right) \cdot Y_{0}\left(\begin{array}{ccc}
l_{1} & \ldots & l_{\kappa} \\
1 & \ldots & \kappa
\end{array}\right) .
\end{aligned}
$$

This identity means that for each summand in the right-hand side of (3.41) we can choose arbitrary permutation of the corresponding sequence $\left(k_{1}, \ldots, k_{\kappa}\right)$. Clearly, the same is true for the corresponding sequence $\left(k_{\kappa+1}, \ldots, k_{n}\right)$.

It follows from (3.5) that

$$
Y_{0}=Y(0, \lambda)=\left(\begin{array}{cc}
I_{\kappa}+o(1) & O\left(\lambda^{-1}\right) \\
O\left(\lambda^{-1}\right) & I_{n-\kappa}+o(1)
\end{array}\right), \quad \text { as } \lambda \rightarrow \infty, \lambda \in S_{p, \varepsilon, R}
$$

Hence if the intersection of the sets $\left\{k_{1}, \ldots, k_{\kappa}\right\}$ and $\{\kappa+1, \ldots, n\}$ consists of $s$ elements, then the corresponding minor $Y_{0}\left(\begin{array}{ccc}k_{1} & \ldots & k_{\kappa} \\ 1 & \ldots & \kappa\end{array}\right)$ contains exactly $s$ lines with entries of the form $O\left(\lambda^{-1}\right)$ while all entries of other lines are of the form $O(1)$. Indeed, if $k_{j}>\kappa$, then $j$ th line of the considered minor coincides with the $\left(k_{j}-\kappa\right)$ th line of the lower-left block of the block-matrix (3.43). Thus, we have

$$
Y_{0}\left(\begin{array}{ccc}
k_{1} & \ldots & k_{\kappa} \\
1 & \ldots & \kappa
\end{array}\right)=O\left(\frac{1}{\lambda^{s}}\right), \quad \lambda \in S_{p, \varepsilon, R}
$$

For the cases $s=0$ and $s=1$ we can obtain sharper estimates. At first, (3.43) directly implies

$$
Y_{0}\left(\begin{array}{lll}
1 & \ldots & \kappa \\
1 & \ldots & \kappa
\end{array}\right)=1+o(1), \quad \text { as } \lambda \rightarrow \infty, \lambda \in S_{p, \varepsilon, R}
$$


Next, assume that $s=1$, i.e. the set $\left\{k_{1}, \ldots, k_{\kappa}\right\}$ is obtained from $\{1, \ldots, \kappa\}$ by replacing its one entry by an entry from $\{\kappa+1, \ldots, n\}$. Assume that $j$ is replaced by $k$, where $1 \leqslant j \leqslant \kappa<k \leqslant n$. Then, according to (3.36), $\operatorname{Re}\left(i b_{k} \lambda\right)>0>$ $\operatorname{Re}\left(i b_{j} \lambda\right)$ and, by (3.5),

$$
\begin{aligned}
& Y_{0}\left(\begin{array}{lllllll}
1 & \ldots & j-1 & k & j+1 & \ldots & \kappa \\
1 & \ldots & j-1 & j & j+1 & \ldots & \kappa
\end{array}\right) \\
& =\operatorname{det}\left(\begin{array}{ccccccc}
1+o(1) & \cdots & o(1) & o(1) & o(1) & \cdots & o(1) \\
\vdots & \ddots & \vdots & \vdots & \vdots & \ddots & \vdots \\
o(1) & \cdots & 1+o(1) & o(1) & o(1) & \cdots & o(1) \\
O\left(\lambda^{-1}\right) & \cdots & O\left(\lambda^{-1}\right) & \frac{r_{k j}(0)+o(1)}{\lambda} & O\left(\lambda^{-1}\right) & \cdots & O\left(\lambda^{-1}\right) \\
o(1) & \cdots & o(1) & o(1) & 1+o(1) & \cdots & o(1) \\
\vdots & \ddots & \vdots & \vdots & \vdots & \ddots & \vdots \\
o(1) & \cdots & o(1) & o(1) & o(1) & \cdots & 1+o(1)
\end{array}\right) \\
& =\frac{r_{k j}(0)+o(1)}{\lambda}, \quad \text { as } \lambda \rightarrow \infty, \lambda \in S_{p, \varepsilon, R},
\end{aligned}
$$

where we set for brevity

$$
r_{j k}(x):=\frac{b_{j} q_{j k}(x)}{b_{j}-b_{k}} .
$$

Further, according to (3.6)

$$
Y_{1}=Y(1, \lambda)=\left(\begin{array}{cc}
I_{\kappa}+o(1) & O\left(\lambda^{-1}\right) \\
O\left(\lambda^{-1}\right) & I_{n-\kappa}+o(1)
\end{array}\right) \cdot E(\lambda), \quad \text { as } \lambda \rightarrow \infty, \lambda \in S_{p, \varepsilon, R},
$$

where

$$
E(\lambda):=\operatorname{diag}\left(e^{i b_{1} \lambda}, \ldots, e^{i b_{n} \lambda}\right) .
$$

Let the set $\left\{k_{\kappa+1}, \ldots, k_{n}\right\}$ contain exactly $s$ entries from the set $\{1, \ldots, \kappa\}$. Then repeating the above reasoning to $Y_{1}$ in place of $Y_{0}$ yields

$$
Y_{1}\left(\begin{array}{ccc}
k_{\kappa+1} & \ldots & k_{n} \\
\kappa+1 & \ldots & n
\end{array}\right)=O\left(\frac{1}{\lambda^{s}}\right) e^{i \tau_{p} \lambda}, \quad \lambda \in S_{p, \varepsilon, R} .
$$


Further, it is easily seen that

$$
Y_{1}\left(\begin{array}{ccc}
\kappa+1 & \ldots & n \\
\kappa+1 & \ldots & n
\end{array}\right)=(1+o(1)) \cdot e^{i \tau_{p} \lambda}
$$

and

$$
Y_{1}\left(\begin{array}{ccccccc}
\kappa+1 & \ldots & k-1 & j & k+1 & \ldots & n \\
\kappa+1 & \ldots & k-1 & k & k+1 & \ldots & n
\end{array}\right)=\left(r_{j k}(1)+o(1)\right) \cdot \frac{e^{i \tau_{p} \lambda}}{\lambda}
$$

as $\lambda \rightarrow \infty, \lambda \in S_{p, \varepsilon, R}$, where $j \in\{1, \ldots, \kappa\}$ and $k \in\{\kappa+1, \ldots, n\}$.

Inserting formulas (3.44) and (3.48) into (3.41) and using (3.42) we get

$$
\begin{aligned}
& \gamma_{p}^{-1} \Delta_{\tilde{Y}}(\lambda) \\
& =\left(J\left(\begin{array}{cccccc}
1 & \ldots & \kappa & \kappa+1 & \ldots & n \\
1 & \ldots & \kappa & n+\kappa+1 & \ldots & n+n
\end{array}\right) \cdot Y_{0}\left(\begin{array}{ccc}
1 & \ldots & \kappa \\
1 & \ldots & \kappa
\end{array}\right) \cdot Y_{1}\left(\begin{array}{ccc}
\kappa+1 & \ldots & n \\
\kappa+1 & \ldots & n
\end{array}\right)\right. \\
& +\sum_{j=1}^{\kappa} \sum_{k=\kappa+1}^{n} J\left(\begin{array}{llllllllll}
1 & \ldots & j-1 & j & j+1 & \ldots & \kappa & \kappa+1 & \ldots & n \\
1 & \ldots & j-1 & k & j+1 & \ldots & \kappa & n+\kappa+1 & \ldots & n+n
\end{array}\right) \cdot Y_{0}\left(\begin{array}{ccccccc}
1 & \ldots & j-1 & k & j+1 & \ldots & \kappa \\
1 & \ldots & j-1 & j & j+1 & \ldots & \kappa
\end{array}\right) \\
& \times Y_{1}\left(\begin{array}{ccc}
\kappa+1 & \ldots & n \\
\kappa+1 & \ldots & n
\end{array}\right) \\
& +\sum_{j=1}^{\kappa} \sum_{k=\kappa+1}^{n} J\left(\begin{array}{cccccccccc}
1 & \ldots & \kappa & \kappa+1 & \ldots & k-1 & k & k+1 & \ldots & n \\
1 & \ldots & \kappa & n+\kappa+1 & \ldots & n+k-1 & n+j & n+k+1 & \ldots & n+n
\end{array}\right) \\
& \left.\times Y_{0}\left(\begin{array}{ccc}
1 & \ldots & \kappa \\
1 & \ldots & \kappa
\end{array}\right) \cdot Y_{1}\left(\begin{array}{ccccccc}
\kappa+1 & \ldots & k-1 & j & k+1 & \ldots & n \\
\kappa+1 & \ldots & k-1 & k & k+1 & \ldots & n
\end{array}\right)\right) \\
& +O\left(\frac{1}{\lambda^{2}}\right) e^{i \tau_{p} \lambda}, \quad \lambda \in S_{p, \varepsilon, R}
\end{aligned}
$$

Let $z_{p}$ be some fixed point in $S_{p, \varepsilon}$. Then it is clear from inequalities (3.36) and definition of matrices $T_{i z_{p} B}(C, D), T_{i z_{p} B}^{c_{j} \rightarrow c_{k}}$ and $T_{i z_{p} B}^{d_{k} \rightarrow d_{j}}$ that

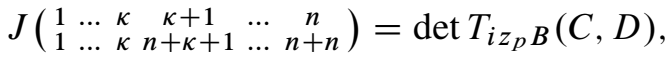

$$
\begin{aligned}
& J\left(\begin{array}{cccccccccc}
1 & \ldots & j-1 & j & j+1 & \ldots & \kappa & \kappa+1 & \ldots & n \\
1 & \ldots & j-1 & k & j+1 & \ldots & \kappa & n+\kappa+1 & \ldots & n+n
\end{array}\right)=\operatorname{det} T_{i z_{p} B}^{c_{j} \rightarrow c_{k}},
\end{aligned}
$$

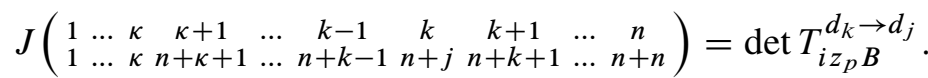


Now inserting (3.45), (3.46), (3.49), (3.50) and (3.52a), (3.52b), (3.52c) into (3.51) we get

$$
\begin{aligned}
\gamma_{p}^{-1} \Delta_{\tilde{Y}}(\lambda) & \\
= & \operatorname{det} T_{i z_{p} B}(C, D) \cdot(1+o(1)) \cdot(1+o(1)) \cdot e^{i \tau_{p} \lambda} \\
& +\sum_{j=1}^{\kappa} \sum_{k=\kappa+1}^{n} \operatorname{det} T_{i z_{p} B}^{c_{j} \rightarrow c_{k}} \cdot \frac{r_{k j}(0)+o(1)}{\lambda} \cdot(1+o(1)) \cdot e^{i \tau_{p} \lambda} \\
& +\sum_{j=1}^{\kappa} \sum_{k=\kappa+1}^{n} \operatorname{det} T_{i z_{p} B}^{d_{k} \rightarrow d_{j}} \cdot(1+o(1)) \cdot \frac{r_{j k}(1)+o(1)}{\lambda} \cdot e^{i \tau_{p} \lambda} \\
& +O\left(\frac{1}{\lambda^{2}}\right) e^{i \tau_{p} \lambda} \\
= & e^{i \tau_{p} \lambda} \cdot\left(\omega_{0}\left(z_{p}\right) \cdot(1+o(1))+o\left(\lambda^{-1}\right)\right. \\
& \left.\quad+\sum_{j=1}^{\kappa} \sum_{k=\kappa+1}^{n} \frac{\operatorname{det} T_{i z_{p} B}^{c_{j} \rightarrow c_{k}} b_{k} q_{k j}(0)-\operatorname{det} T_{i z_{p} B}^{d_{k} \rightarrow d_{j}} b_{j} q_{j k}(1)}{\lambda\left(b_{k}-b_{j}\right)}\right),
\end{aligned}
$$

as $\lambda \rightarrow \infty, \lambda \in S_{p, \varepsilon, R}$. Rewriting the double sum in the last equality with account of (3.36) we arrive at formula (3.14) with the required form of $\omega_{1}\left(z_{p}\right)$.

\section{Explicit completeness results}

4.1. Explicit sufficient conditions of completeness. Now we are ready to state our main result on completeness of the root vectors of the boundary value problem (1.2)-(1.4) in terms of the matrices $B, C, D$ and $Q(\cdot)$.

Theorem 4.1. Assume that $Q(\cdot) \in L^{1}\left([0,1] ; \mathbb{C}^{n \times n}\right)$ and $q_{j k}$ is continuous at points 0 and 1 if $b_{j} \neq b_{k}$. Let $\omega_{0}\left(z_{k}\right)$ and $\omega_{1}\left(z_{k}\right)$ be given by (3.17) and (3.18), respectively. Assume also that there exist three admissible complex numbers $z_{1}, z_{2}, z_{3}$ satisfying the following conditions:

(a) the origin is an interior point of the triangle $\triangle_{z_{1} z_{2} z_{3}}$;

(a) $\left|\omega_{0}\left(z_{k}\right)\right|+\left|\omega_{1}\left(z_{k}\right)\right| \neq 0, k \in\{1,2,3\}$.

Then the system of root functions of the BVP (1.2)-(1.4) is complete and minimal in $L^{2}\left([0,1] ; \mathbb{C}^{n}\right)$. 
Remark 4.2. Note that $\omega_{j}(\cdot), j \in\{0,1\}$, is a constant function in each sector $\sigma_{k}, k \in\{1, \ldots, m\}$, introduced before formula (1.7). Hence $\omega_{j}(\cdot), j \in\{0,1\}$, is piecewise constant function in the plane $\mathbb{C}$ with cuts along the lines $\partial \sigma_{k}, k \in$ $\{1, \ldots, m\}$. It is easily seen that the assumptions of Theorem 4.1 fail if and only if both $\omega_{0}(\cdot)$ and $\omega_{1}(\cdot)$ vanish in the open half-plane $\{\lambda \in \mathbb{C}: \operatorname{Re}(c \lambda)>0\}$ for some $c \neq 0$.

Proof of Theorem 4.1. Recall that the lines

$$
l_{j}=\left\{\lambda \in \mathbb{C}: \operatorname{Re}\left(i b_{j} \lambda\right)=0\right\}
$$

divide the complex plane into $m$ sectors $\sigma_{1}, \ldots, \sigma_{m}$. Let $k \in\{1,2,3\}$ be fixed. Note that the point $z_{k}$ can be not feasible but it is clear from definition of $\omega_{0}(\cdot)$ and $\omega_{1}(\cdot)$ that they are constant in each sector $\sigma_{j}$. Hence if $z_{k}$ is not feasible, that is, it lies at one of the lines

$$
l_{j k}=\left\{\lambda \in \mathbb{C}: \operatorname{Re}\left(i b_{j} \lambda\right)=\operatorname{Re}\left(i b_{k} \lambda\right)\right\},
$$

we can replace it by any point with arbitrary close argument to make it feasible and to conserve the condition (a) of the theorem. Thus, we can assume that the points $z_{1}, z_{2}, z_{3}$ are feasible. Then combining condition (b) of the theorem with Proposition 3.4 implies for $k \in\{1,2,3\}$

$$
\begin{aligned}
|\Delta(\lambda)| & \geqslant C\left|\omega_{0}\left(z_{k}\right)+\frac{\omega_{1}\left(z_{k}\right)}{\lambda}\right| e^{\operatorname{Re}\left(i \tau_{k} \lambda\right)} \\
& \geqslant C_{1} \frac{e^{\operatorname{Re}\left(i \tau_{k} \lambda\right)}}{|\lambda|}, \quad|\lambda|>R, \arg \lambda=\arg z_{k},
\end{aligned}
$$

where $C, C_{1}>0$,

$$
\tau_{k}:=\sum_{\operatorname{Re}\left(i b_{j} z_{k}\right)>0} b_{j},
$$

and $R$ is sufficiently large. To complete the proof it remains to apply Theorem 2.3.

The following result is easily derived from Theorem 4.1 (cf. [37, Corolary 3.2]).

Corollary 4.3. Let $Q$ satisfy assumptions of Theorem 4.1, and let $\left|\omega_{0}( \pm z)\right|+$ $\left|\omega_{1}( \pm z)\right| \neq 0$ for some admissible number $z$. Then the system of root functions of the BVP (1.2)-(1.4) is complete and minimal in $L^{2}\left([0,1] ; \mathbb{C}^{n}\right)$. 
Remark 4.4. In connection with Theorem 4.1 we mention the fundamental paper [51] by A.A. Shkalikov, where he studied BVP for ODE (1.1) with spectral parameter in boundary conditions. In particular, the notion of weakly $B$-regular boundary conditions might be treated as an analogue of the notion of normal BVP of order 0 from [51], while conditions of Theorem 4.1 correlate with those of normal BVP of order 1 from [51]. Moreover, it is proved in [51] that the system of root functions of the linearization of the normal BVP for ODE (1.1) is complete in certain direct sums of Sobolev spaces. For certain matrices $B=\operatorname{diag}\left(b_{1}, \ldots, b_{n}\right)$ with simple spectrum this result correlate with [37, Theorem 1.2] and Theorem 4.1.

We first apply Theorem 4.1 to $2 \times 2$ case. Let

$$
\left(\begin{array}{ll}
C & D
\end{array}\right)=\left(\begin{array}{llll}
a_{11} & a_{12} & a_{13} & a_{14} \\
a_{21} & a_{22} & a_{23} & a_{24}
\end{array}\right),
$$

and

$$
J_{j k}:=\operatorname{det}\left(\begin{array}{ll}
a_{1 j} & a_{1 k} \\
a_{2 j} & a_{2 k}
\end{array}\right), \quad j, k \in\{1, \ldots, 4\} .
$$

Proposition 4.5. Let $n=2, \arg b_{1} \neq \arg b_{2}$, and let $q_{12}, q_{21}$ be continuous at the endpoints 0 and 1 . Then the system of root functions of the boundary value problem (1.2)-(1.4) is complete and minimal in $L^{2}\left([0,1] ; \mathbb{C}^{2}\right)$ whenever

$$
\left|J_{32}\right|+\left|b_{1} J_{13} q_{12}(0)+b_{2} J_{42} q_{21}(1)\right| \neq 0,
$$

and

$$
\left|J_{14}\right|+\left|b_{1} J_{13} q_{12}(1)+b_{2} J_{42} q_{21}(0)\right| \neq 0 \text {. }
$$

Proof. Since $\arg b_{1} \neq \arg b_{2}$ then there exists $z \in \mathbb{C}$ such that $\operatorname{Re}\left(i b_{1} z\right)<0<$ $\operatorname{Re}\left(i b_{2} z\right)$. Then, in accordance with definition of $J_{j k}$ and the numbers $\omega_{0}(z), \omega_{1}(z)$,

$$
\omega_{0}(z)=J_{14}, \quad \omega_{1}(z)=\frac{J_{24} b_{1} q_{21}(0)-J_{13} b_{1} q_{12}(1)}{b_{1}-b_{2}},
$$

and

$$
\omega_{0}(-z)=J_{32}, \quad \omega_{1}(-z)=\frac{J_{31} b_{2} q_{12}(0)-J_{42} b_{2} q_{21}(1)}{b_{2}-b_{1}} .
$$

Conditions (4.2), (4.3) imply $\left|\omega_{0}( \pm z)\right|+\left|\omega_{1}( \pm z)\right| \neq 0$. Hence Corollary 4.3 yields the result. 
Remark 4.6. In the case of $2 \times 2$ Dirac-type systems $\left(b_{1}<0<b_{2}\right)$ this result improves Theorem 5.1 from [37] where the completeness was proved under the stronger assumption $q_{12}, q_{21} \in C^{1}[0,1]$ while was stated for $q_{12}, q_{21} \in$ $C[0,1]$. It happened because the precise version of Lemma 5.4 from [37] requires a stronger assumption $Q(\cdot) \in C^{1}\left([0,1] ; \mathbb{C}^{n \times n}\right)$ instead of $Q(\cdot) \in C\left([0,1] ; \mathbb{C}^{n \times n}\right)$ (cf. [34, Theorem 1.1]). In our forthcoming paper the completeness property of BVP (1.2)-(1.4) for $2 \times 2$ Dirac-type systems will be discussed in detail.

In the case $b_{2} b_{1}^{-1} \notin \mathbb{R}$ Proposition 4.5 improves Theorems 1.4 and 1.6 from [1] where the completeness property was proved for analytic $Q(\cdot)$.

The next result demonstrates that Theorem 4.1 cannot be treated as a perturbation result since unperturbed operator $L_{C, D}(0)$ may have incomplete system of root functions.

Corollary 4.7. Let $\kappa \in\{1, \ldots, n-1\}, \operatorname{Re} b_{j}<0$ for $j \in\{1, \ldots, \kappa\}, \operatorname{Re} b_{j}>0$ for $j \in\{\kappa+1, \ldots, n\}$, and the first boundary condition in (1.4) is of the form $y_{1}(0)=0$. Then the following holds.

(i) Assume that $Q$ is continuous at the endpoints 0 and 1 of the segment $[0,1]$,

$$
\operatorname{det} T_{B}(C, D) \neq 0 \text { and } \sum_{j=\kappa+1}^{n} \frac{\operatorname{det} T_{-B}^{c_{j} \rightarrow c_{1}}}{b_{1}-b_{j}} \cdot q_{1 j}(0) \neq 0 .
$$

Then the system of root functions of the operator $L_{C, D}(Q)$ is complete and minimal in $L^{2}\left([0,1] ; \mathbb{C}^{n}\right)$.

(ii) If $q_{1 j}(x)=0$ for $x \in[0, \varepsilon], j \in\{2, \ldots, n\}$, for some $\varepsilon>0$, then the system of root functions of the operator $L_{C, D}(Q)$ is incomplete in $L^{2}\left([0,1] ; \mathbb{C}^{n}\right)$ and its defect is infinite. In particular, the latter is valid for the operator $L_{C, D}(0)$ with zero potential.

Proof. (i) The condition $y_{1}(0)=0$ means that $c_{11}=1, c_{1 k}=0$ for $k \in\{2, \ldots, n\}$, and $d_{1 k}=0, k \in\{1, \ldots, n\}$. Therefore, the matrix $T_{-B}(C, D)$ has zero first line and hence $\omega_{0}(i)=0$. Moreover, due to the structure of the first row of $\left(\begin{array}{ll}C & D\end{array}\right)$, $\operatorname{det} T_{-B}^{d_{k} \rightarrow d_{j}}=0, j, k \in\{1, \ldots, n\}$, and $\operatorname{det} T_{-B}^{c_{j} \rightarrow c_{k}}=0$, for $k>1$. Now the assumption on $\operatorname{Re} b_{j}$, definition of $\omega_{1}(\cdot)$, and condition (4.4) together imply

$$
\omega_{1}(i)=\sum_{j=\kappa+1}^{n} \frac{\operatorname{det} T_{-B}^{c_{j} \rightarrow c_{1}} \cdot b_{1} q_{1 j}(0)}{b_{1}-b_{j}} \neq 0 .
$$

Due to the first relation in (4.4) $\omega_{0}(-i)=\operatorname{det} T_{B}(C, D) \neq 0$. It remains to apply Corollary 4.3. 
(ii) Under our assumption each solution $y=\operatorname{col}\left(y_{1}, \ldots, y_{n}\right)$ of the problem (1.2)-(1.4) satisfies

$$
y_{1}^{\prime}=i b_{1} \lambda y_{1}+i b_{1} q_{11}(x) y_{1}, \quad x \in[0, \varepsilon], \quad \text { and } \quad y_{1}(0)=0 .
$$

By the uniqueness theorem, $y_{1}(x)=0$ for $x \in[0, \varepsilon]$. Hence each

$$
f=\operatorname{col}\left(f_{1}, 0, \ldots, 0\right) \in L^{2}\left([0,1] ; \mathbb{C}^{n}\right)
$$

with $f_{1}$ vanishing on $[\varepsilon, 1]$ is orthogonal to the system of root functions of the operator $L_{C, D}(Q)$.

Remark 4.8. Let $n=3, \kappa=1$ and $y_{1}(0)=0$. Then condition (4.4) takes the form

$$
\left|\begin{array}{ll}
d_{22} & d_{23} \\
d_{32} & d_{33}
\end{array}\right| \neq 0 \text { and } \quad\left|\begin{array}{ll}
d_{21} & c_{23} \\
d_{31} & c_{33}
\end{array}\right| \cdot \frac{q_{12}(0)}{b_{2}-b_{1}}+\left|\begin{array}{ll}
d_{21} & c_{22} \\
d_{31} & c_{32}
\end{array}\right| \cdot \frac{q_{13}(0)}{b_{1}-b_{3}} \neq 0
$$

Therefore, if $\left|q_{12}(0)\right|+\left|q_{13}(0)\right| \neq 0$, then, in general, the system of root functions of the operator $L_{C, D}(Q)$ with the first boundary condition $y_{1}(0)=0$, is complete in $L^{2}\left([0,1] ; \mathbb{C}^{3}\right)$.

Finally, we specify Corollary 4.3 for $4 \times 4$ Dirac type equation subject to special boundary conditions. This statement will be applied in Section 6 for study of the Timoshenko beam model.

Corollary 4.9. Let $n=4, B=\operatorname{diag}\left(-b_{1}, b_{1},-b_{2}, b_{2}\right)$, where $b_{1}, b_{2}>0$, let $Q \in$ $L^{1}\left([0,1] ; \mathbb{C}^{4 \times 4}\right)$, where $Q$ is continuous at the endpoints 0 and 1 , and matrices $C$ and $D$ are of the form

$$
C=\left(\begin{array}{llll}
1 & 1 & 0 & 0 \\
0 & 0 & 0 & 0 \\
0 & 0 & 1 & 1 \\
0 & 0 & 0 & 0
\end{array}\right), \quad D=\left(\begin{array}{cccc}
0 & 0 & 0 & 0 \\
d_{1} & d_{2} & 0 & 0 \\
0 & 0 & 0 & 0 \\
0 & 0 & d_{3} & d_{4}
\end{array}\right)
$$

Assume that

$$
\left|d_{2} d_{4}\right|+\left|d_{1} d_{4} q_{12}(1)\right|+\left|d_{2} d_{3} q_{34}(1)\right| \neq 0
$$

and

$$
\left|d_{1} d_{3}\right|+\left|d_{2} d_{3} q_{21}(1)\right|+\left|d_{1} d_{4} q_{43}(1)\right| \neq 0 \text {. }
$$

Then the system of root functions of the BVP (1.2)-(1.4) is complete and minimal in $L^{2}\left([0,1] ; \mathbb{C}^{4}\right)$. 
Proof. By the definition of the matrix $T_{B}(C, D)$,

$$
T_{B}(C, D)=\left(\begin{array}{cccc}
1 & 0 & 0 & 0 \\
0 & d_{2} & 0 & 0 \\
0 & 0 & 1 & 0 \\
0 & 0 & 0 & d_{4}
\end{array}\right)
$$

and hence

$$
\omega_{0}(-i)=\operatorname{det} T_{B}(C, D)=d_{2} d_{4}
$$

In our case the double sum in (3.18) for $\omega_{1}(-i)$ involves only values $j=1,3$ and $k=2$, 4. It follows from definition of matrices $T_{i z B}^{c_{j} \rightarrow c_{k}}$ and $T_{i z B}^{d_{k} \rightarrow d_{j}}$ that

$$
\begin{array}{ll}
\operatorname{det} T_{B}^{c_{1} \rightarrow c_{2}}=d_{2} d_{4}, & \operatorname{det} T_{B}^{d_{2} \rightarrow d_{1}}=d_{1} d_{4}, \\
\operatorname{det} T_{B}^{c_{1} \rightarrow c_{4}}=0, & \operatorname{det} T_{B}^{d_{4} \rightarrow d_{1}}=0, \\
\operatorname{det} T_{B}^{c_{3} \rightarrow c_{2}}=0, & \operatorname{det} T_{B}^{d_{2} \rightarrow d_{3}}=0, \\
\operatorname{det} T_{B}^{c_{3} \rightarrow c_{4}}=d_{2} d_{4}, & \operatorname{det} T_{B}^{d_{4} \rightarrow d_{3}}=d_{2} d_{3} .
\end{array}
$$

Inserting these expressions into (3.18) we obtain

$$
\omega_{1}(-i)=\frac{1}{2}\left(d_{2} d_{4} q_{21}(0)+d_{1} d_{4} q_{12}(1)+d_{2} d_{4} q_{43}(0)+d_{2} d_{3} q_{34}(1)\right) .
$$

Note that if $d_{2}=0$, then $\omega_{1}(-i)=\frac{1}{2} d_{1} d_{4} q_{12}(1)$. On the other hand, if $d_{4}=$ 0 , then $\omega_{1}(-i)=\frac{1}{2} d_{2} d_{3} q_{34}(1)$. This allows us to rewrite condition $\left|\omega_{0}(-i)\right|+$ $\left|\omega_{1}(-i)\right| \neq 0$ in the form of the first relation in (4.7).

Similarly, one verifies that condition $\left|\omega_{0}(i)\right|+\left|\omega_{1}(i)\right| \neq 0$ turns into the second relation in (4.7). One completes the proof by applying Corollary 4.3.

The following simple lemma will be useful for us in Section 6.

Lemma 4.10. Condition (4.7) is fulfilled if and only if each of the following conditions is satisfied

$$
\begin{array}{rr}
\left|d_{1}\right|+\left|d_{2}\right| \neq 0, & \left|d_{3}\right|+\left|d_{4}\right| \neq 0, \\
\left|d_{1}\right|+\left|d_{3}\right| \neq 0, & \left|d_{2}\right|+\left|d_{4}\right| \neq 0, \\
\left|d_{1}\right|+\left|q_{21}(1)\right| \neq 0, & \left|d_{2}\right|+\left|q_{12}(1)\right| \neq 0, \\
\left|d_{3}\right|+\left|q_{43}(1)\right| \neq 0, & \left|d_{4}\right|+\left|q_{34}(1)\right| \neq 0 .
\end{array}
$$


Proof. If $d_{1} d_{2} d_{3} d_{4} \neq 0$ then the statement is obvious. Further assume that $d_{j}=0$ for some $j \in\{1,2,3,4\}$. Let for definiteness, $d_{1}=0$. Then conditions (4.10)-(4.13) are satisfied if and only if

$$
d_{2} d_{3} q_{21}(1) \neq 0 \quad \text { and } \quad\left|d_{4}\right|+\left|q_{34}(1)\right| \neq 0
$$

This, in turn, is equivalent to (4.7) whenever $d_{1}=0$, and we are done.

4.2. Example. Here we illustrate Proposition 4.5 by investigation of the completeness and minimality of the system of vector functions

$$
\left\{\left(\begin{array}{c}
e^{a n x} \sin n x \\
n e^{a n x}(\sin n x+i \cos n x)
\end{array}\right)\right\}_{n \in \mathbb{Z} \backslash\{0\}}, \quad a \in \mathbb{C},
$$

in the space $L^{2}\left([0, \pi] ; \mathbb{C}^{2}\right)$.

\section{Corollary 4.11. Let}

$$
\text { i } a \notin(-\infty,-1] \cap[1, \infty) \text {. }
$$

Then system (4.14) is complete and minimal in $L^{2}\left([0, \pi] ; \mathbb{C}^{2}\right)$.

Proof. Since $a \neq \pm i$ there exists $\theta \in \mathbb{C} \backslash\{\pi n\}_{n \in \mathbb{Z}}$ such that $a=\operatorname{ctg} \theta$. Consider the following boundary value problem

$$
\begin{gathered}
\left\{\begin{array}{l}
y_{1}^{\prime}=e^{i \theta} \lambda y_{1}+y_{2}, \\
y_{2}^{\prime}=e^{-i \theta} \lambda y_{2},
\end{array}\right. \\
y_{1}(0)=y_{1}(1)=0 .
\end{gathered}
$$

Straightforward calculation shows that its spectrum is simple, consists of the eigenvalues $\left\{\frac{\pi n}{\sin \theta}\right\}_{n \in \mathbb{Z} \backslash\{0\}}$, and the system of the corresponding eigenfunctions is

$$
\left\{\left(\begin{array}{l}
e^{a \pi n x} \sin \pi n x \\
\pi n \cdot e^{(a-i) \pi n x}
\end{array}\right)\right\}_{n \in \mathbb{Z} \backslash\{0\}}
$$

It is easily seen that a potential matrix of the operator $L_{C, D}(Q)$ associated with the boundary value problem (4.16a)-(4.16b) is constant: $Q(\cdot)=\left(\begin{array}{cc}0 & -e^{-i \theta} \\ 0 & 0\end{array}\right)$. Clearly,

$$
B=\operatorname{diag}\left(b_{1}, b_{2}\right):=-i \operatorname{diag}\left(e^{i \theta}, e^{-i \theta}\right) .
$$

Moreover, due to (4.15) $\arg b_{1} \neq \arg b_{2}$. 
Clearly, boundary conditions (4.16b) imply $J_{13}=1$, while the other determinants $J_{j k}$ are zero. In particular, boundary conditions (4.16b) are non-weakly regular and even degenerate: $\Delta_{0}(\cdot) \equiv 0$. However, conditions (4.2)-(4.3) take now the form $q_{12}(0) q_{12}(1) \neq 0$ and clearly, are fulfilled. Hence, by Proposition 4.5, the system of eigenvectors (4.17) is complete and minimal in $L^{2}\left([0,1] ; \mathbb{C}^{2}\right)$. The latter is equivalent to the completeness and minimality of the system (4.14) in $L^{2}\left([0, \pi] ; \mathbb{C}^{2}\right)$.

Remark 4.12. In connection with Corollary 4.11 let us consider one more system of functions $\mathcal{K}_{a}=\left\{e^{a n x} \sin n x\right\}_{n \in \mathbb{Z} \backslash\{0\}}$. Clearly, it is a system of the eigenfunctions of the problem

$$
y^{\prime \prime}-2 a \lambda y^{\prime}+\left(a^{2}+1\right) \lambda^{2} y=0, \quad y(0)=y(\pi)=0 .
$$

It is known (see [26, Part II, Appendix A1], [32] and the references therein) that this system is twofold complete in $L^{2}[0, \pi]$ in the sense of M.V. Keldysh [21]. The latter means completeness of the system $\left\{\operatorname{col}\left(e^{a n x} \sin n x, n e^{a n x} \sin n x\right)\right\}_{n \in \mathbb{Z} \backslash\{0\}}$ in $L^{2}\left([0, \pi] ; \mathbb{C}^{2}\right)$. So, the statement of Corollary 4.11 is in a sense close to the twofold completeness and minimality of the system $\mathcal{K}_{a}$. Note that investigation of the completeness and basis property of a "half" system

$$
\mathcal{K}_{a}^{+}:=\left\{e^{a n x} \sin n x\right\}_{n=1}^{\infty}
$$

in $L^{2}[0, \pi]$ has been initiated by A.G. Kostyuchenko and constitutes his named problem.

Note also that in the case $a \in \mathbb{R}$ problem (4.18) naturally arises in the investigation of the solvability of the following elliptic boundary value problem in the strip $\Omega=[0, \pi] \times \mathbb{R}_{+}$:

$$
\begin{cases}L u:=\frac{\partial^{2} u}{\partial x^{2}}-2 a \frac{\partial^{2} u}{\partial x \partial t}+\left(a^{2}+1\right) \frac{\partial^{2} u}{\partial t^{2}}=0, & \\ u(0, t)=u(\pi, t)=0, & t \geqslant 0, \\ u(x, 0)=u_{0}(x), & u_{0} \in L^{2}[0, \pi] .\end{cases}
$$

Since equation $L u=0$ is elliptic, the Cauchy problem in the strip is incorrect. Applying the Fourier method, i.e. seeking for a solution of (4.19) in the form $u(x, t)=e^{\lambda t} y(x)$, leads to problem (4.18). 
4.3. Necessary conditions of completeness. Next we present some necessary conditions of completeness.

Proposition 4.13. Let boundary conditions (1.4) be of the form $y(0)=A y(1)$, where $\operatorname{det} A \neq 0$,

$$
A B+B A=0 \quad \text { and } \quad Q(1-x)=A^{-1} Q(x) A, \quad x \in[0, \varepsilon] \text {, for some } \varepsilon>0 \text {. }
$$

Then the defect of the system of root functions of the operator

$$
L:=L_{C, D}(Q)
$$

in $L^{2}\left([0,1] ; \mathbb{C}^{n}\right)$ is infinite.

Proof. Let $\lambda$ be an eigenvalue of $L$ and let $\left\{u_{p}(x)\right\}_{p=1}^{m}$ be a chain of the eigenfunction and associated functions of the operator $L$ corresponding to $\lambda$. Put $u_{0}(x):=$ 0 . It is clear that $u_{p}(\cdot), p \in\{0,1, \ldots, m\}$, satisfies boundary conditions (1.4) and the following identity holds

$$
\operatorname{Lu}_{p}(x)=\lambda u_{p}(x)+u_{p-1}(x), \quad x \in[0,1], p \in\{1, \ldots, m\} .
$$

Denote $v_{p}(x):=A u_{p}(1-x)$. Let us prove by induction that $u_{p}(x)=v_{p}(x)$, $x \in[0, \varepsilon]$. For $p=0$ it is clear. Let $p>0$. It follows from (4.21) and (1.2) that

$$
\begin{aligned}
\left(u_{p}^{\prime}\right)(1-x) & =i B(\lambda-Q(1-x)) u_{p}(1-x)+i B u_{p-1}(1-x) \\
& =i B\left[(\lambda-Q(1-x)) A^{-1} v_{p}(x)+A^{-1} v_{p-1}(x)\right] .
\end{aligned}
$$

Further, combining relations (4.20), (4.22) with the definition of $v_{p}$ and taking into account induction hypothesis yields

$$
\begin{aligned}
L v_{p}(x) & =-i B^{-1} v_{p}^{\prime}(x)+Q(x) v_{p}(x) \\
& =i B^{-1} A \cdot\left(u_{p}^{\prime}\right)(1-x)+Q(x) v_{p}(x) \\
& =-i A B^{-1} i B\left[(\lambda-Q(1-x)) A^{-1} v_{p}(x)+A^{-1} v_{p-1}(x)\right]+Q(x) v_{p}(x) \\
& =\lambda v_{p}(x)+v_{p-1}(x)+\left(Q(x)-A Q(1-x) A^{-1}\right) v_{p}(x) \\
& =\lambda v_{p}(x)+u_{p-1}(x), \quad x \in[0, \varepsilon] .
\end{aligned}
$$

Next, due to the assumption, $v_{p}(0)=A u_{p}(1)=u_{p}(0)$. Thus, both $u_{p}$ and $v_{p}$ satisfy the same non-homogenous linear equation (4.21) for $x \in[0, \varepsilon]$ as well as the same initial condition at zero. Therefore, by the Cauchy uniqueness theorem,

$$
u_{p}(x)=v_{p}(x)=A u_{p}(1-x), \quad x \in[0, \varepsilon] .
$$


Further, let $f \in L^{2}\left([0,1] ; \mathbb{C}^{n}\right)$ and let

$$
f(x)=0 \quad \text { for } x \in[\varepsilon, 1-\varepsilon],
$$

and

$$
f(1-x)=-A^{*} f(x), \quad \text { for } x \in[0, \varepsilon] .
$$

Then one has for $p \geqslant 0$

$$
\begin{aligned}
\int_{0}^{1}\left\langle u_{p}(x), f(x)\right\rangle d x & =\int_{0}^{\varepsilon}\left\langle u_{p}(x), f(x)\right\rangle d x+\int_{0}^{\varepsilon}\left\langle u_{p}(1-x), f(1-x)\right\rangle d x \\
& =\int_{0}^{\varepsilon}\left(\left\langle u_{p}(x), f(x)\right\rangle+\left\langle A^{-1} u_{p}(x),-A^{*} f(x)\right\rangle\right) d x \\
& =0 .
\end{aligned}
$$

This identity shows that each vector-function $f$ satisfying (4.23) is orthogonal to the system of root functions of the operator $L_{C, D}(Q)$. This completes the proof.

Note that existence of a nonsingular solution of the matrix equation

$$
A B+B A=0
$$

is equivalent to the similarity of the matrices $B$ and $-B$ :

$$
A B A^{-1}=-B \text {. }
$$

The latter amounts to saying that the spectra $\sigma(B)$ and $\sigma(-B)$ coincide with their multiplicities. Since $B$ is diagonal, we can restate Proposition 4.13 as follows.

Corollary 4.14. Let $n=2 p$ and $B=\operatorname{diag}(\widetilde{B},-\widetilde{B})$, where

$$
\widetilde{B}=\operatorname{diag}\left(I_{n_{1}} b_{1}, \ldots, I_{n_{r}} b_{r}\right), \quad n_{1}+\cdots+n_{r}=p .
$$

Further, let

$$
A=\left(\begin{array}{cc}
0 & A_{1} \\
A_{2} & 0
\end{array}\right), \quad A_{j}=\operatorname{diag}\left(A_{j 1}, \ldots, A_{j r}\right), \quad A_{j k} \in \mathrm{GL}\left(n_{k}, \mathbb{C}\right), \quad j \in\{1,2\},
$$

let boundary conditions (1.4) be of the form $y(0)=A y(1)$, and let

$$
Q(1-x)=A^{-1} Q(x) A, \quad x \in[0, \varepsilon], \text { for some } \varepsilon>0 .
$$

Then the system of root functions of the operator $L_{C, D}(Q)$ is incomplete in the space $L^{2}\left([0,1] ; \mathbb{C}^{n}\right)$ and its defect is infinite. 
Proof. Due to the block structure of the matrices $\widetilde{B}, A_{1}$ and $A_{2}$, one has $A B+$ $B A=0$. Since $A_{j k}$ is nonsingular, $\operatorname{det} A \neq 0$. Therefore, Proposition 4.13 completes the result.

Remark 4.15. Note that in the case of $2 \times 2$ Dirac system $(B=\operatorname{diag}(-1,1)$, $\left.q_{11} \equiv q_{22} \equiv 0\right)$ Proposition 4.13 turns into [37, Proposition 5.12]. Indeed, consider $2 \times 2$ Dirac equation subject to the boundary conditions $y_{1}(0)=\alpha_{1} y_{2}(1)$, $y_{2}(0)=\alpha_{2} y_{1}(1)$. Setting $A=\left(\begin{array}{cc}0 & \alpha_{1} \\ \alpha_{2} & 0\end{array}\right)$, one rewrites these conditions as $y(0)=$ $A y$ (1). Moreover, condition (4.24) turns into $\alpha_{1} q_{21}(1-x)=\alpha_{2} q_{12}(x), x \in$ $[0, \varepsilon] \cap[1-\varepsilon, 1]$, for some $\varepsilon>0$, i.e. coincides with the respective condition from [37]. Similar result for Sturm-Liouville operator subject to degenerate boundary conditions was proved earlier in [35].

\section{The Riesz basis property for root functions}

Here we investigate the Riesz basis property for operator $L_{C, D}(Q)$ by reduction it to the operator $L_{\widetilde{C}, \widetilde{D}}(\widetilde{Q})$ being a perturbation of a normal operator. To this end we find conditions for matrices $C$ and $D$ guarantying that $L_{C, D}(0)$ is normal.

Lemma 5.1. (i) An operator

$$
L:=L_{C, D}(0)
$$

is normal if and only if

$$
C B C^{*}=D B D^{*} .
$$

(ii) Boundary conditions (1.4) are regular, i.e. $\operatorname{det} T_{i z B}(C, D) \neq 0$ for each admissible $z$, whenever (5.1) is fulfilled.

(iii) If $Q \in L^{1}\left([0,1] ; \mathbb{C}^{n \times n}\right)$ and condition (5.1) is satisfied, then the system of root functions of the operator $L_{C, D}(Q)$ is complete and minimal in $L^{2}\left([0,1] ; \mathbb{C}^{n}\right)$.

Proof. (i) It is easily seen that

$$
L L^{*} y=L^{*} L y=-\left(B B^{*}\right)^{-1} y^{\prime \prime}
$$

for $y \in W^{2,2}\left([0,1] ; \mathbb{C}^{n}\right)$. Therefore, $L$ is normal if and only if $\operatorname{dom}(L)=\operatorname{dom}\left(L^{*}\right)$, which is equivalent to $(L f, g)=\left(f, L^{*} g\right), f, g \in \operatorname{dom}(L)$. In turn, integrating by parts one gets that this identity is equivalent to

$$
\left\langle B^{-1} f(0), g(0)\right\rangle=\left\langle B^{-1} f(1), g(1)\right\rangle, \quad f, g \in \operatorname{dom}(L) .
$$


Put

$$
\widetilde{B}:=\operatorname{diag}\left(B^{-1},-B^{-1}\right)
$$

and equip the space $\mathcal{H}=\mathbb{C}^{n} \oplus \mathbb{C}^{n}$ with the bilinear form

$$
w(u, v):=\langle\widetilde{B} u, v\rangle=\left\langle B^{-1} u_{1}, v_{1}\right\rangle-\left\langle B^{-1} u_{2}, v_{2}\right\rangle,
$$

where $u=\operatorname{col}\left(u_{1}, u_{2}\right)$ and $v=\operatorname{col}\left(v_{1}, v_{2}\right)$. Now condition (5.2) takes the form

$$
w(u, v)=0, \quad u, v \in \mathcal{H}_{1}:=\operatorname{ker}\left(\begin{array}{ll}
C & D
\end{array}\right):=\left\{\operatorname{col}\left(u_{1}, u_{2}\right): C u_{1}+D u_{2}=0\right\} .
$$

On the other hand, the equality $C B C^{*}=D B D^{*}$ can be rewritten as

$$
\left\langle B^{-1} B C^{*} h, B C^{*} k\right\rangle=\left\langle B^{-1}\left(-B D^{*} h\right),-B D^{*} k\right\rangle, \quad h, k \in \mathbb{C}^{n} .
$$

Using (5.3) one rewrites this equality in the form

$$
w(u, v)=0, \quad u, v \in \mathcal{H}_{2}:=\left\{\operatorname{col}\left(B C^{*} h,-B D^{*} h\right): h \in \mathbb{C}^{n}\right\} .
$$

Thus, to prove the statement it suffices to show that (5.4) is equivalent to (5.5). To this end we prove that $\mathcal{H}_{1}$ is the right $w$-orthogonal complement of $\mathcal{H}_{2}$,

$$
\mathcal{H}_{1}=\mathcal{H}_{2}^{[\perp]}:=\left\{u \in \mathcal{H}: w(v, u)=0, v \in \mathcal{H}_{2}\right\} .
$$

Indeed, if $v=\operatorname{col}\left(B C^{*} h,-B D^{*} h\right) \in \mathcal{H}_{2}$ and $u=\operatorname{col}\left(u_{1}, u_{2}\right) \in \mathcal{H}$, then $w(v, u)=\left\langle B^{-1}\left(B C^{*}\right) h, u_{1}\right\rangle-\left\langle B^{-1}\left(-B D^{*}\right) h, u_{2}\right\rangle=\left\langle h, C u_{1}+D u_{2}\right\rangle, \quad h \in \mathbb{C}^{n}$. It follows that $w(v, u)=0$ for each $v \in \mathcal{H}_{2}$ if and only if $C u_{1}+D u_{2}=0$, i.e. $u \in \mathcal{H}_{1}$. Next, maximality condition (1.6) yields $\operatorname{dim} \mathcal{H}_{1}=\operatorname{dim} \mathcal{H}_{2}=n$.

Now, if (5.5) is satisfied, then $\mathcal{H}_{2} \subset \mathcal{H}_{2}^{[\perp]}=\mathcal{H}_{1}$. Since $\operatorname{dim} \mathcal{H}_{1}=\operatorname{dim} \mathcal{H}_{2}$, one has $\mathcal{H}_{1}=\mathcal{H}_{2}$ and (5.4) is fulfilled. The opposite implication is derived similarly.

(ii) Since $L=L_{C, D}(0)$ is normal, (5.4) is satisfied. Let $\beta_{1}^{-1}, \beta_{2}^{-1}, \ldots, \beta_{2 n}^{-1}$ be the eigenvalues of $\widetilde{B}$ and let $e_{1}, e_{2}, \ldots, e_{2 n}$ be the corresponding normalized eigenvectors. Note that $\beta_{k}=-\beta_{n+k}=b_{k}, k \in\{1, \ldots, n\}$. For every admissible $z$, i.e. for $z$ satisfying $\operatorname{Re}\left(i z b_{k}\right) \neq 0, k \in\{1, \ldots, n\}$, we put

$$
\mathcal{H}_{z}:=\operatorname{span}\left\{e_{k}: \operatorname{Re}\left(i z \beta_{k}\right)>0\right\} .
$$

Since $\beta_{n+k}=-\beta_{k}, k \in\{1, \ldots, n\}$, then $\operatorname{dim} \mathcal{H}_{z}=n$ for every admissible $z$. Next we note that $T_{i z B}(C, D)=\left.\left(\begin{array}{ll}C & D\end{array}\right)\right|_{\mathcal{H}_{z}}$. Therefore, $\operatorname{det} T_{i z B}(C, D) \neq 0$ if and only if $\operatorname{ker}\left(\begin{array}{ll}C & D\end{array}\right) \cap \mathcal{H}_{z}=\{0\}$. Let $u \in \mathcal{H}_{z}$. Then $u=\sum_{\operatorname{Re}\left(i z \beta_{k}\right)>0} c_{k} e_{k}$, and

$$
\operatorname{Re}(i z\langle u, \widetilde{B} u\rangle)=\sum_{\operatorname{Re}\left(i z \beta_{k}\right)>0}\left|c_{k}\right|^{2} \operatorname{Re}\left(i z \overline{\beta_{k}^{-1}}\right)=\sum_{\operatorname{Re}\left(i z \beta_{k}\right)>0} \frac{\left|c_{k}\right|^{2}}{\left|\beta_{k}\right|^{2}} \operatorname{Re}\left(i z \beta_{k}\right) .
$$


Hence

$$
\operatorname{Re}(i z\langle u, \widetilde{B} u\rangle)>0, \quad u \in \mathcal{H}_{z} \backslash\{0\} .
$$

On the other hand, due to (5.4), $\langle u, \widetilde{B} u\rangle=\overline{\langle\widetilde{B} u, u\rangle}=0$ for $u \in \operatorname{ker}\left(\begin{array}{ll}C & D\end{array}\right)$.

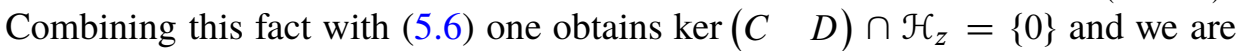
done.

(iii) It follows from (ii) that boundary conditions (1.4) are weakly $B$-regular. The completeness and minimality of the root functions of the operator $L_{C, D}(Q)$ is implied by [37, Theorem 1.2].

Remark 5.2. Let $Q \in L^{2}\left([0,1] ; \mathbb{C}^{n \times n}\right)$. Then (unbounded) multiplication operator $Q: f \rightarrow Q(x) f, f \in L^{2}\left([0,1] ; \mathbb{C}^{n}\right)$, is relatively compact with respect to $L_{C, D}(0)$. Therefore statement (iii) is implied by the classical Keldysh theorem (cf. [39, Theorem 4.3]) if in addition the spectrum of $L_{C, D}(0)$ lies on the union of rays $\left\{\lambda \in \mathbb{C}: \arg \lambda=\varphi_{k}\right\}, k \in\{1, \ldots, n\}$.

Recall the following definitions from [18] and [39].

Definition 5.3. (i) A sequence $\left\{f_{k}\right\}_{k=1}^{\infty}$ of vectors in $\mathfrak{H}$ is called a Riesz basis if it admits a representation $f_{k}=T e_{k}, k \in \mathbb{N}$, where $\left\{e_{k}\right\}_{k=1}^{\infty}$ is an orthonormal basis in $\mathfrak{H}$ and $T: \mathfrak{H} \rightarrow \mathfrak{H}$ is a bounded operator with bounded inverse.

(ii) A sequence of subspaces $\left\{\mathfrak{H}_{k}\right\}_{k=1}^{\infty}$ is called a Riesz basis of subspaces in $\mathfrak{H}$ if there exists a complete sequence of mutually orthogonal subspaces $\left\{\mathfrak{H}_{k}^{\prime}\right\}_{k=1}^{\infty}$ and a bounded operator $T$ in $\mathfrak{H}$ with bounded inverse such that $\mathfrak{H}_{k}=T \mathfrak{H}_{k}^{\prime}, k \in \mathbb{N}$.

(iii) A sequence $\left\{f_{k}\right\}_{k=1}^{\infty}$ of vectors in $\mathfrak{H}$ is called a Riesz basis with parentheses if each its finite subsequence is linearly independent, and there exists an increasing sequence $\left\{n_{k}\right\}_{k=0}^{\infty} \subset \mathbb{N}$ such that $n_{0}=1$ and the sequence

$$
\mathfrak{H}_{k}:=\operatorname{span}\left\{f_{j}\right\}_{j=n_{k-1}}^{n_{k}-1},
$$

forms a Riesz basis of subspaces in $\mathfrak{H}$. Subspaces $\mathfrak{H}_{k}$ are called blocks.

To state the next result we need the following definition.

Definition 5.4. Let $\left\{\varphi_{k}\right\}_{k=1}^{n}$ be a sequence of angles, $\varphi_{k} \in(-\pi, \pi]$, and $\varepsilon>0$. Numbers $\lambda, \mu \in \mathbb{C}$ are called $\varepsilon$-close with respect to $\left\{\varphi_{k}\right\}_{k=1}^{n}$, if, for some $k \in$ $\{1, \ldots, n\}$,

$$
\lambda, \mu \in\left\{z \in \mathbb{C}:\left|\arg z-\varphi_{k}\right|<\varepsilon\right\}
$$

and

$$
\left|\operatorname{Re}\left(e^{-i \varphi_{k}}(\lambda-\mu)\right)\right|<\varepsilon .
$$


In other words, $\lambda$ and $\mu$ are $\varepsilon$-close if for some $k$ they belong to a small angle with the bisectrix

$$
l_{+}\left(\varphi_{k}\right):=\left\{\lambda \in \mathbb{C}: \arg \lambda=\varphi_{k}\right\}
$$

and their projections on this ray are close.

Let $A$ be an operator with compact resolvent and let $\Omega$ be a bounded subset of $\mathbb{C}$. We put

$$
N(\Omega, A):=\sum_{\lambda \in \sigma(A) \cap \Omega} m_{a}(\lambda, A)=\sum_{\lambda \in \sigma(A) \cap \Omega} \operatorname{dim} \mathcal{R}_{\lambda}(A) .
$$

Our investigation of the Riesz basis property of the operator $L_{C, D}$ is based on the following statement that can easily be extracted from [40] and [39, §I.6].

Proposition 5.5. Let $\mathfrak{H}$ be a separable Hilbert space and let $G$ be a normal operator with compact resolvent in $\mathfrak{H}$. Assume that the spectrum of $G$ lies on the union of rays $l_{+}\left(\varphi_{1}\right), \ldots, l_{+}\left(\varphi_{n}\right)$, and

$$
\sup _{z \in \mathbb{C}} N(\mathbb{D}(z), G)<\infty, \quad \mathbb{D}(z):=\{\zeta \in \mathbb{C}:|\zeta-z|<1\} .
$$

Finally, let $T$ be a bounded operator in $\mathfrak{H}$ and let $\varepsilon>0$ be arbitrarily small. Then the system of root vectors of the operator $A=G+T$ forms a Riesz basis with parentheses in $\mathfrak{H}$, where each block is constituted by the root subspaces corresponding to the eigenvalues of $A$ that are mutually $\varepsilon$-close with respect to the sequence $\left\{\varphi_{k}\right\}_{k=1}^{n}$.

Proof. Since $T$ is bounded, it is relatively compact with respect to $G$. Hence by [39, Corollary 3.7], all but finitely many eigenvalues of $A=G+T$ belong to the union of non-overlapping sectors

$$
\Omega_{j}(\varepsilon):=\left\{\lambda \in \mathbb{C}:\left|\arg \lambda-\varphi_{j}\right|<\varepsilon\right\}, \quad j \in\{1, \ldots, n\} .
$$

Fix $j \in\{1, \ldots, n\}$ and set

$$
G_{j}:=e^{-i \varphi_{j}} G .
$$

Condition (5.7) implies condition (6.21) of [39, Lemma 6.8],

$$
\sup _{k \in \mathbb{N}} N\left(\left(r_{k}-q r_{k}^{p}, r_{k}+q r_{k}^{p}\right), G_{j}\right)<\infty,
$$

with $p=0$, any $q>0$ and any increasing sequence $\left\{r_{k}\right\}_{k=1}^{\infty}$. Let $\left\{\lambda_{j, k}\right\}_{k=1}^{\infty}$ be the sequence of eigenvalues of $A$ belonging to $\Omega_{j}(\varepsilon)$ and ordered in ascending order of $\operatorname{Re}\left(e^{-i \varphi_{j}} \lambda_{j, k}\right)$. Put

$$
r_{k}:=\operatorname{Re}\left(e^{-i \varphi_{j}} \lambda_{j, k}\right)-\varepsilon / 2, \quad k \in \mathbb{N} .
$$


Applying [39, Lemma 6.8] to the operator $G_{j}$ with $p=0, q=\|T\|+4 \varepsilon$ and the above sequence $\left\{r_{k}\right\}_{k=1}^{\infty}$, we conclude that there exists $x_{k} \in\left(r_{k}-\varepsilon / 2, r_{k}+\varepsilon / 2\right)$, $k \in \mathbb{N}$, such that the sequence $\left\{x_{k}\right\}_{k=1}^{\infty}$ is strictly monotone and the sequence of subspaces

$$
\mathfrak{H}_{j, k}:=\operatorname{span}\left\{\mathcal{R}_{\lambda_{j, s}}(A): x_{k} \leqslant \operatorname{Re}\left(e^{-i \varphi_{j}} \lambda_{j, s}\right)<x_{k+1}\right\}, \quad k \in \mathbb{N},
$$

forms a Riesz basis of subspaces in its closed linear span. It follows from definition of $r_{k}$ and $x_{k}$ that

$$
\operatorname{Re}\left(e^{-i \varphi_{j}} \lambda_{j, k}\right)-\varepsilon<x_{k}<\operatorname{Re}\left(e^{-i \varphi_{j}} \lambda_{j, k}\right), \quad k \in \mathbb{N} .
$$

Hence root subspaces of $A$ corresponding to the eigenvalues of $A$, that are not $\varepsilon$-close with respect to $\left\{\varphi_{k}\right\}_{k=1}^{n}$, belong to different blocks. Let $\lambda_{1}^{\prime}, \ldots, \lambda_{m}^{\prime}$ be the sequence of eigenvalues of $A$ not belonging to the union of sectors $\cup_{j=1}^{n} \Omega_{j}(\varepsilon)$. Clearly, the family of subspaces

$$
\left\{\mathcal{R}_{\lambda_{k}^{\prime}}\right\}_{k=1}^{m},\left\{\mathfrak{H}_{1, k}\right\}_{k=1}^{\infty}, \ldots,\left\{\mathfrak{H}_{n, k}\right\}_{k=1}^{\infty},
$$

forms a Riesz basis of subspaces in its closed linear span. Since the latter spans the system of root vectors of the operator $A$, the Keldysh theorem (cf. [39, Theorem 4.3]) yields its completeness in $\mathfrak{H}$. Therefore, the system of root vectors of the operator $A$ forms a Riesz basis with parentheses having the required properties of the blocks.

Now we are ready to prove our main result on the Riesz basis property of BVP (1.2)-(1.4).

Theorem 5.6. Let $Q \in L^{\infty}\left([0,1] ; \mathbb{C}^{n \times n}\right)$ and

$$
B=\operatorname{diag}\left(B_{j}\right)_{j=1}^{r}, \quad C=\operatorname{diag}\left(C_{j}\right)_{j=1}^{r}, \quad D=\operatorname{diag}\left(D_{j}\right)_{j=1}^{r},
$$

where

$$
B_{j}=\left(\begin{array}{cc}
b_{j 1} I_{n_{j}} & 0 \\
0 & b_{j 2} I_{n_{j}}
\end{array}\right), \quad b_{j 1} b_{j 2}^{-1} \in(-\infty, 0)
$$

and

$$
C_{j}=\left(\begin{array}{cc}
C_{j 1} & C_{j 2} \\
0 & 0
\end{array}\right), \quad D_{j}=\left(\begin{array}{cc}
0 & 0 \\
D_{j 1} & D_{j 2}
\end{array}\right), \quad C_{j 1}, C_{j 2}, D_{j 1}, D_{j 2} \in \mathrm{GL}\left(n_{j}, \mathbb{C}\right) .
$$


Then the system of root functions of the operator $A:=L_{C, D}(Q)$ forms a Riesz basis with parentheses in $L^{2}\left([0,1] ; \mathbb{C}^{n}\right)$, where each block is constituted by the root subspaces corresponding to the eigenvalues of $A$ that are mutually $\varepsilon$-close with respect to the sequence of angles $\left\{-\varphi_{1}, \ldots,-\varphi_{r}, \pi-\varphi_{1}, \ldots, \pi-\varphi_{r}\right\}$. Here $\varphi_{j}=\arg \left(b_{j 1}-b_{j 2}\right), j \in\{1, \ldots, r\}$, and $\varepsilon>0$ is sufficiently small.

Proof. First we show that the operator $L_{C, D}(Q)$ is similar to the operator $L_{\widetilde{C}, \tilde{D}}(\tilde{Q})$ with the same matrix $B$ and matrices $\widetilde{C}, \widetilde{D}$ satisfying (5.1). To this end we use the gauge transform $W: y \rightarrow W(x) y$, with $W(\cdot)$ satisfying

$$
W(x) B=B W(x), \quad x \in[0,1],
$$

and

$$
W(\cdot) \in C^{1}\left([0,1] ; \mathbb{C}^{n \times n}\right), \quad W^{-1}(\cdot) \in C\left([0,1] ; \mathbb{C}^{n \times n}\right) .
$$

Then the operator $L_{C, D}(Q)$ is transformed into the operator

$$
L_{\tilde{C}, \tilde{D}}(\tilde{Q})=W^{-1} L_{C, D}(Q) W
$$

with the same $B$, and matrices $\widetilde{C}, \widetilde{D}, \widetilde{Q}(\cdot)$ given by

$$
\begin{aligned}
\widetilde{C} & :=C W(0), \\
\widetilde{D} & :=D W(1), \\
\widetilde{Q}(x) & :=W^{-1}(x) Q(x) W(x)-i W^{-1}(x) B^{-1} W^{\prime}(x) .
\end{aligned}
$$

Since $W(\cdot), W^{\prime}(\cdot), W^{-1}(\cdot), Q(\cdot) \in L^{\infty}\left([0,1] ; \mathbb{C}^{n \times n}\right)$, then $\widetilde{Q} \in L^{\infty}\left([0,1] ; \mathbb{C}^{n \times n}\right)$.

Due to the block diagonal structure (5.10)-(5.11) of the matrices $B, C_{j}$, and $D_{j}$, we can choose $W_{0}, W_{1} \in \mathrm{GL}(n, \mathbb{C})$ such that

$$
W_{k} B=B W_{k}, \quad k \in\{0,1\},
$$

and

$$
\begin{array}{ll}
C W_{0}=\operatorname{diag}\left(\widetilde{C}_{j}\right)_{j=1}^{r}, & \widetilde{C}_{j}:=\left(\begin{array}{cc}
I_{n_{j}} & b_{j} I_{n_{j}} \\
0 & 0
\end{array}\right), \quad b_{j}:=\left(-b_{j 1} b_{j 2}^{-1}\right)^{1 / 2}, \\
D W_{1}=\operatorname{diag}\left(\widetilde{D}_{j}\right)_{j=1}^{r}, \quad \widetilde{D}_{j}:=\left(\begin{array}{cc}
0 & 0 \\
I_{n_{j}} & b_{j} I_{n_{j}}
\end{array}\right), \quad j \in\{1, \ldots, r\} .
\end{array}
$$

Choose any branch of logarithm and put

$$
\tilde{W}:=\log \left(W_{0}^{-1} W_{1}\right) .
$$


Clearly, $\tilde{W}$ is well defined since the matrix $W_{0}^{-1} W_{1}$ is nonsingular. Hence

$$
W(x):=W_{0} e^{x \tilde{W}}
$$

satisfies $(5.12 \mathrm{a}),(5.12 \mathrm{~b})$, and $W(0)=W_{0}, W(1)=W_{1}$. Define a gauge transform $W: y \rightarrow W(x) y$. In view of (5.13)-(5.14b) the matrices $\widetilde{C}, \widetilde{D}$ of the new operator $L_{\widetilde{C}, \widetilde{D}}(\widetilde{Q})=W^{-1} L_{C, D}(Q) W$ are $\widetilde{C}=\operatorname{diag}\left(\widetilde{C}_{j}\right)_{j=1}^{r}$ and $\widetilde{D}=\operatorname{diag}\left(\widetilde{D}_{j}\right)_{j=1}^{r}$ where $\widetilde{C}_{j}$ and $\widetilde{D}_{j}$ are given by $(5.14 \mathrm{a})$ and $(5.14 \mathrm{~b})$, respectively.

Straightforward calculation shows that

$$
\widetilde{C}_{j} B_{j} \widetilde{C}_{j}^{*}=\widetilde{D}_{j} B_{j} \widetilde{D}_{j}^{*}=0, \quad j \in\{1, \ldots, r\} .
$$

Hence

$$
\widetilde{C} B \widetilde{C}^{*}=\widetilde{D} B \widetilde{D}^{*}=0 .
$$

By Lemma 5.1, the operator

$$
G:=L_{\tilde{C}, \widetilde{D}^{(0)}}
$$

is normal. Its spectrum coincides with the set of zeros of the characteristic determinant $\Delta(\cdot)=\operatorname{det}(\widetilde{C}+\widetilde{D} \widetilde{\Phi}(1, \cdot))$. The fundamental matrix $\widetilde{\Phi}(\cdot, \lambda)$ of the operator $L_{\widetilde{C}, \widetilde{D}}(0)$ is $\widetilde{\Phi}(x, \lambda)=e^{i B \lambda x}$. Hence, in view of the block-diagonal structure of the matrices $B, \widetilde{C}, \widetilde{D}$, we obtain

$$
\begin{aligned}
\Delta(\lambda)=\prod_{j=1}^{r} \operatorname{det}\left(\widetilde{C}_{j}+\widetilde{D}_{j} e^{i B_{j} \lambda}\right) & =\prod_{j=1}^{r} \operatorname{det}\left(\begin{array}{cc}
I_{n_{j}} & b_{j} I_{n_{j}} \\
e^{i b_{j 1} \lambda} I_{n_{j}} & b_{j} e^{i b_{j 2} \lambda} I_{n_{j}}
\end{array}\right) \\
& =\prod_{j=1}^{r}\left(b_{j}^{n_{j}} \cdot\left(e^{i b_{j 2} \lambda}-e^{i b_{j 1} \lambda}\right)^{n_{j}}\right) .
\end{aligned}
$$

Hence

$$
\sigma(G)=\left\{\frac{2 \pi k}{b_{j 1}-b_{j 2}}: k \in \mathbb{Z}, j \in\{1, \ldots, r\}\right\} .
$$

Thus $\sigma(G)$ lies on the union of rays $\left\{l_{+}\left(-\varphi_{j}\right)\right\}_{1}^{r}$ and $\left\{l_{+}\left(\pi-\varphi_{j}\right)\right\}_{1}^{r}$, where $\varphi_{j}=$ $\arg \left(b_{j 1}-b_{j 2}\right), j \in\{1, \ldots, r\}$. Moreover, $\sigma(G)$ is the union of a finite number of arithmetic progressions and multiplicities of eigenvalues are bounded, hence condition (5.7) is satisfied. Since $\widetilde{Q}(\cdot)$ is bounded, then, by Proposition 5.5, the system of root functions of the operator

$$
\widetilde{A}:=L_{\tilde{C}, \widetilde{D}}(\tilde{Q})=L_{\tilde{C}, \widetilde{D}}(0)+\widetilde{Q}=G+\widetilde{Q}
$$

forms a Riesz basis with parentheses in $\mathfrak{H}$, where each block is constituted by the root subspaces corresponding to the mutually close eigenvalues of $A$ in the sense of Definition 5.4. Since $A=L_{C, D}(Q)$ is similar to $\tilde{A}$, the same is true for the root functions of the operator $L_{C, D}(Q)$. 
As a consequence of this result we obtain the Riesz basis property of the system of root functions for Dirac system with general splitting boundary conditions.

Corollary 5.7. Let $n=2 m, B=\operatorname{diag}\left(b_{1} I_{m}, b_{2} I_{m}\right), b_{1}<0<b_{2}$, and $Q \in$ $L^{\infty}\left([0,1] ; \mathbb{C}^{n \times n}\right)$, and let

$$
C=\left(\begin{array}{cc}
C_{1} & C_{2} \\
0 & 0
\end{array}\right), \quad D=\left(\begin{array}{cc}
0 & 0 \\
D_{1} & D_{2}
\end{array}\right), \quad C_{1}, C_{2}, D_{1}, D_{2} \in \operatorname{GL}(m, \mathbb{C})
$$

Then the system of root functions of the operator $L_{C, D}(Q)$ forms a Riesz basis with parentheses in $L^{2}\left([0,1] ; \mathbb{C}^{n \times n}\right)$.

Remark 5.8. In connection with Corollary 5.7 we mention recent paper [44] where it is proved the Bari-Markus property for spectral projections of Dirac operator $\left(-b_{1}=b_{2}=1\right)$ with a potential $Q \in L^{2}\left([0,1] ; \mathbb{C}^{n}\right)$, and $C_{1}=C_{2}=D_{1}=$ $D_{2}=I_{m}$.

Similarly to Theorem 5.6 we can obtain the following result.

Proposition 5.9. Let

$$
B=\operatorname{diag}\left(b_{1} I_{n_{1}}, \ldots, b_{r} I_{n_{r}}\right), \quad n=n_{1}+\cdots+n_{r},
$$

and

$$
C=\operatorname{diag}\left(C_{j}\right)_{j=1}^{r}, \quad D=\operatorname{diag}\left(D_{j}\right)_{j=1}^{r}, \quad C_{j}, D_{j} \in G L\left(n_{j}, \mathbb{C}\right), j \in\{1, \ldots, r\},
$$

and $Q \in L^{\infty}\left([0,1] ; \mathbb{C}^{n \times n}\right)$. Then the system of root functions of the operator

$$
A:=L_{C, D}(Q)
$$

forms a Riesz basis with parentheses in $L^{2}\left([0,1] ; \mathbb{C}^{n}\right)$, where each block is constituted by the root subspaces corresponding to the eigenvalues of $A$ that are mutually $\varepsilon$-close with respect to the sequence of angles $\left\{-\varphi_{1}, \ldots,-\varphi_{r}, \pi-\varphi_{1}, \ldots, \pi-\varphi_{r}\right\}$. Here $\varphi_{j}=\arg b_{j}, j \in\{1, \ldots, r\}$, and $\varepsilon>0$ is sufficiently small.

Proof. The proof is similar to that of Theorem 5.6. At first choosing an appropriate gauge transform, we transform the operator $L_{C, D}(Q)$ into $L_{\widetilde{C}, \widetilde{D}}(\widetilde{Q})$ with $\widetilde{C}_{j}=$ $\widetilde{D}_{j}=I_{n_{j}}$. It follows that the operator $G:=L_{\widetilde{C}, \widetilde{D}}(0)$ is normal and its spectrum is of the form $\sigma(G)=\left\{2 \pi k / b_{j}: k \in \mathbb{Z}, j \in\{1, \ldots, r\}\right\}$. Hence the same argument as in the proof of Theorem 5.6 yields the result. 
A direct consequence of this result is the Riesz basis property of the periodic (reps. antiperiodic) $B V P$ with general matrix $B$.

Corollary 5.10. Let $B=\operatorname{diag}\left(b_{1}, \ldots, b_{n}\right) \in \mathrm{GL}(n, \mathbb{C}), C= \pm D=I_{n}$ and $Q \in L^{\infty}\left([0,1] ; \mathbb{C}^{n \times n}\right)$. Then the system of root functions of the operator $L_{C, D}(Q)$ forms a Riesz basis with parentheses in $L^{2}\left([0,1] ; \mathbb{C}^{n \times n}\right)$.

Remark 5.11. In the case of Dirac type systems $\left(B=B^{*}\right)$ we can extend the statements of Theorem 5.6 and Proposition 5.9 to the case of $Q \in L^{2}\left([0,1] ; \mathbb{C}^{n \times n}\right)$. Indeed, it suffices to apply Theorem 2 from the recent paper [52] instead of the quoted results from [40] and [39, §I.6]. Note however, that in [52, Theorem 2] only the basis property instead of the Riesz basis property was stated.

Remark 5.12. The Riesz basis property for $2 \times 2$ Dirac equation subject to splitting boundary conditions has been investigated in numerous papers [58, 20, 6, 7]. The most general statement covering Corollary 5.7 (for $n=1$ ) was obtained by Djakov and Mityagin [7] who relaxed the assumption on a potential matrix to $Q \in L^{2}\left([0,1] ; \mathbb{C}^{2}\right)$. Moreover, these authors proved in [7] the Riesz basis property for $2 \times 2$ Dirac equation subject to general strictly regular boundary conditions.

For $2 \times 2$ Dirac system Corollary 5.10 was proved in [6] under weaker assumption $Q \in L^{2}\left([0,1] ; \mathbb{C}^{2}\right)$. Moreover, these authors found out [11] a criterion for the system of root functions of the periodic boundary value problem for $2 \times 2$ Dirac equation to contain a Riesz basis (without parentheses). Similar result for Sturm-Liouville operator $-\frac{d^{2}}{d x^{2}}+q$ was obtained by different methods in $[15,16]$ and [11]. Both criteria are formulated directly in terms of periodic and Dirichlet eigenvalues. Moreover, in [9, Theorem 13], [11, Theorem 19] (see also [8]) it is established criteria for eigenfunctions and associated functions to form a Riesz basis for periodic 1D Dirac operator (resp. Sturm-Liouville operator) in terms of the Fourier coefficients of $Q$ (resp. $q$ ). Equivalence of this formulation to that in terms of periodic and Dirichlet eigenvalues is explained in [11, Theorem 24]. Let us mention in this connection the paper [53] where Riesz basis property for periodic Sturm-Liouville operator was obtained under certain explicit sufficient conditions in terms of Fourier coefficients of a potential $q$.

In the simplest case $B=I_{n}$ we can indicate a criterion for the system of root functions of the operator $L_{C, D}(Q)$ to form a Riesz basis with parentheses.

Corollary 5.13. Let $B=I_{n}$ and $Q \in L^{1}\left([0,1] ; \mathbb{C}^{n \times n}\right)$. Then the system of root functions of the operator $L_{C, D}(Q)$ forms a Riesz basis with parentheses in $L_{2}\left([0,1] ; \mathbb{C}^{n}\right)$ if and only if $\operatorname{det}(C \cdot D) \neq 0$. 
Proof. Applying the gauge transform $y \rightarrow W(x) y$ with $W(\cdot)$ described in the beginning of the proof of Proposition 3.4, we see that the operator $L_{C, D}(Q)$ is similar to the operator $L_{\widetilde{C}, \widetilde{D}}(0)$ with $\widetilde{C}=C, \widetilde{D}=D W(1)$ and zero potential matrix. Further, since $B=I_{n}$, then $T_{B}(\widetilde{C}, \widetilde{D})=D W(1)$ and $T_{-B}(\tilde{C}, \widetilde{D})=C$. Hence, $\operatorname{det}(C \cdot D) \neq 0$ if and only if $\operatorname{det} T_{B}(\widetilde{C}, \widetilde{D}) \cdot \operatorname{det} T_{-B}(\widetilde{C}, \widetilde{D}) \neq 0$. Therefore, by [37, Proposition 4.6], the system of root functions of the operator $L_{\tilde{C}, \widetilde{D}}(0)$ has infinite defect, whenever $\operatorname{det}(C \cdot D)=0$. On the other hand, if $\operatorname{det}(C \cdot D) \neq 0$ then, by Proposition 5.9, applied with $r=1$ and $Q=0$, the system of root functions of the operator $L_{\widetilde{C}, \widetilde{D}}(0)$ forms a Riesz basis with parentheses. Similarity of the operators $L_{C, D}(Q)$ and $L_{\widetilde{C}, \widetilde{D}}(0)$ completes the proof.

\section{Application to the Timoshenko beam model}

Here we obtain some important geometric properties of the system of root functions for the dynamic generator of the Timoshenko beam model. Consider the following linear system of two coupled hyperbolic equations for $t \geqslant 0$

$$
\begin{array}{rlrl}
I_{\rho}(x) \Phi_{t t} & =K(x)\left(W_{x}-\Phi\right)+\left(E I(x) \Phi_{x}\right)_{x}-p_{1}(x) \Phi_{t}, & & x \in[0, \ell], \text { (6.1a) } \\
\rho(x) W_{t t} & =\left(K(x)\left(W_{x}-\Phi\right)\right)_{x}-p_{2}(x) W_{t}, & x \in[0, \ell] . \quad(6.1 \mathrm{~b})
\end{array}
$$

The vibration of the Timoshenko beam of the length $\ell$ clamped at the left end is governed by the system (6.1a)-(6.1b) subject to the following boundary conditions for $t \geqslant 0$ [57]:

$$
\begin{gathered}
W(0, t)=\Phi(0, t)=0, \\
\left.\left(E I(x) \Phi_{x}(x, t)+\alpha_{1} \Phi_{t}(x, t)+\beta_{1} W_{t}(x, t)\right)\right|_{x=l}=0, \\
\left.\left(K(x)\left(W_{x}(x, t)-\Phi(x, t)\right)+\alpha_{2} W_{t}(x, t)+\beta_{2} \Phi_{t}(x, t)\right)\right|_{x=l}=0 .
\end{gathered}
$$

Here $W(x, t)$ is the lateral displacement at a point $x$ and time $t, \Phi(x, t)$ is the bending angle at a point $x$ and time $t, \rho(x)$ is a mass density, $K(x)$ is the shear stiffness of a uniform cross-section, $I_{\rho}(x)$ is the rotary inertia, $E I(x)$ is the flexural rigidity at a point $x, p_{1}(x)$ and $p_{2}(x)$ are locally distributed feedback functions, $\alpha_{j}, \beta_{j} \in \mathbb{C}, j \in\{1,2\}$. Boundary conditions at the right end contain as partial cases most of the known boundary conditions if $\alpha_{1}, \alpha_{2}$ are allowed to be infinity. 
Regarding the coefficients we assume that they satisfy the following general conditions:

$$
\begin{aligned}
\rho, I_{\rho}, K, E I \in C[0, \ell], \quad p_{1}, p_{2} \in L^{1}[0, \ell], \\
0<C_{1} \leqslant \rho(x), I_{\rho}(x), K(x), E I(x) \leqslant C_{2}, \quad x \in[0, \ell] .
\end{aligned}
$$

The energy space associated with the problem (6.1a)-(6.2c) is

$$
\mathfrak{H}:=\widetilde{H}_{0}^{1}[0, \ell] \times L^{2}[0, \ell] \times \tilde{H}_{0}^{1}[0, \ell] \times L^{2}[0, \ell],
$$

where $\tilde{H}_{0}^{1}[0, \ell]:=\left\{f \in W^{1,2}[0, \ell]: f(0)=0\right\}$. The norm in the energy space is defined as follows:

$$
\|y\|_{\mathfrak{H}}^{2}=\int_{0}^{\ell}\left(E I\left|y_{1}^{\prime}\right|^{2}+I_{\rho}\left|y_{2}\right|^{2}+K\left|y_{3}^{\prime}-y_{1}\right|^{2}+\rho\left|y_{4}\right|^{2}\right) d x,
$$

with

$$
y=\operatorname{col}\left(y_{1}, y_{2}, y_{3}, y_{4}\right) .
$$

The problem (6.1a)-(6.2c) can be rewritten as

$$
y_{t}=i \mathcal{L} y,\left.\quad y(x, t)\right|_{t=0}=y_{0}(x),
$$

where $y$ and $\mathcal{L}$ are given by

$$
\begin{gathered}
y=\left(\begin{array}{c}
\Phi(x, t) \\
\Phi_{t}(x, t) \\
W(x, t) \\
W_{t}(x, t)
\end{array}\right), \\
\mathcal{L}\left(\begin{array}{c}
y_{1} \\
y_{2} \\
y_{3} \\
y_{4}
\end{array}\right)=\frac{1}{i}\left(\begin{array}{c}
\frac{1}{y_{\rho}(x)}\left(K(x)\left(y_{3}^{\prime}-y_{1}\right)+\left(E I(x) y_{1}^{\prime}\right)^{\prime}-p_{1}(x) y_{2}\right) \\
y_{4} \\
\frac{1}{\rho(x)}\left(\left(K(x)\left(y_{3}^{\prime}-y_{1}\right)\right)^{\prime}-p_{2}(x) y_{4}\right)
\end{array}\right)
\end{gathered}
$$

on the domain

$$
\begin{aligned}
\operatorname{dom}(\mathcal{L})=\{ & y=\operatorname{col}\left(y_{1}, y_{2}, y_{3}, y_{4}\right): y_{1}, y_{2}, y_{3}, y_{4} \in \tilde{H}_{0}^{1}[0, \ell], \\
& E I \cdot y_{1}^{\prime} \in A C[0, \ell],\left(E I \cdot y_{1}^{\prime}\right)^{\prime}-p_{1} y_{2} \in L^{2}[0, \ell], \\
& K \cdot\left(y_{3}^{\prime}-y_{1}\right) \in A C[0, \ell],\left(K \cdot\left(y_{3}^{\prime}-y_{1}\right)\right)^{\prime}-p_{2} y_{4} \in L^{2}[0, \ell], \quad(6.8) \\
& \left(E I \cdot y_{1}^{\prime}\right)(\ell)+\alpha_{1} y_{2}(\ell)+\beta_{1} y_{4}(\ell)=0, \\
& \left.\left(K \cdot\left(y_{3}^{\prime}-y_{1}\right)\right)(\ell)+\alpha_{2} y_{4}(\ell)+\beta_{2} y_{2}(\ell)=0\right\} .
\end{aligned}
$$


Timoshenko beam model is investigated in numerous papers (see [57, 24, 54, 61, $60,59]$ and the references therein). A number of stability, controllability, and optimization problems were studied. Note also that the general model (6.1a)-(6.2c) of spatially non-homogenous Timoshenko beam with both boundary and locally distributed damping covers the cases studied by many authors. Geometric properties of the system of root functions of the operator $\mathcal{L}$ play important role in investigation of different properties of the problem (6.1a)-(6.2c).

Below we establish completeness and the Riesz basis property with parentheses of the operator $\mathcal{L}$, without analyzing its spectrum. For convenience we impose the following additional algebraic assumption on $\mathcal{L}$ :

$$
v(x):=\frac{E I(x) \rho(x)}{K(x) I_{\rho}(x)}=\text { const }, \quad x \in[0, \ell],
$$

Clearly, (6.9) is satisfied whenever $I_{\rho}(x)=R \rho(x)$, where $R=$ const is a crosssectional area of the beam, $E I$ and $K$ are constant functions, while $\rho \in A C[0, \ell]$ and is arbitrary positive (cf. condition (6.13)). Our approach to the spectral properties of the operator $\mathcal{L}$ is based on the similarity reduction of $\mathcal{L}$ to a special $4 \times 4$ Dirac-type operator. To state the result we need some additional preparations.

Let $\gamma(\cdot)$ be given by

$$
\sqrt{\frac{I_{\rho}(x)}{E I(x)}}=b_{1} \gamma(x), \quad \text { where } b_{1}>0 \text { and } \int_{0}^{\ell} \gamma(x) d x=1 .
$$

Conditions (6.3a) and (6.3b) imply together that $\gamma \in C[0, \ell]$ and is positive. Further, in view of (6.9) we have

$$
\sqrt{\frac{\rho(x)}{K(x)}}=b_{2} \gamma(x), \quad \text { where } b_{2}>0 .
$$

Let

$$
\begin{aligned}
B & :=\operatorname{diag}\left(-b_{1}, b_{1},-b_{2}, b_{2}\right) . \\
\Theta(x) & :=-2 i \operatorname{diag}\left(I_{\rho}(x), I_{\rho}(x), \rho(x), \rho(x)\right), \\
h_{1}(x) & :=\sqrt{E I(x) I_{\rho}(x)}, \quad h_{2}(x):=\sqrt{K(x) \rho(x)} .
\end{aligned}
$$

In the sequel we assume that

$$
h_{1}, h_{2} \in A C[0, \ell]
$$


Therefore, according to (6.3a)-(6.3b) the following matrix function is well-defined:

$$
\hat{Q}(x):=\Theta^{-1}(x)\left(\begin{array}{cccc}
p_{1}+h_{1}^{\prime} & p_{1}-h_{1}^{\prime} & h_{2} & -h_{2} \\
p_{1}+h_{1}^{\prime} & p_{1}-h_{1}^{\prime} & h_{2} & -h_{2} \\
-h_{2} & -h_{2} & p_{2}+h_{2}^{\prime} & p_{2}-h_{2}^{\prime} \\
h_{2} & h_{2} & p_{2}+h_{2}^{\prime} & p_{2}-h_{2}^{\prime}
\end{array}\right) .
$$

Next, we set

$$
t(x)=\int_{0}^{x} \gamma(s) d s, \quad x \in[0, \ell] .
$$

Since $\gamma \in C[0, \ell]$ and is positive, the function $t(\cdot)$ strictly increases on $[0, \ell]$, $t(\cdot) \in C^{1}[0, \ell]$, and due to $(6.10) t(\ell)=1$. Hence, the inverse function

$$
x(\cdot):=t^{-1}(\cdot)
$$

is well defined, strictly increasing on $[0,1]$, and $x(\cdot) \in C^{1}[0,1]$. Next, we put

$$
Q(t):=\hat{Q}(x(t))=:\left(q_{j k}(t)\right)_{j, k=1}^{4}, \quad t \in[0,1] .
$$

Finally, let

$$
\begin{aligned}
C & =\left(\begin{array}{llll}
1 & 1 & 0 & 0 \\
0 & 0 & 0 & 0 \\
0 & 0 & 1 & 1 \\
0 & 0 & 0 & 0
\end{array}\right), \\
D & =\left(\begin{array}{cccc}
0 & 0 & 0 & 0 \\
\alpha_{1}-h_{1}(\ell) & \alpha_{1}+h_{1}(\ell) & \beta_{1} & \beta_{1} \\
0 & 0 & 0 & 0 \\
\beta_{2} & \beta_{2} & \alpha_{2}-h_{2}(\ell) & \alpha_{2}+h_{2}(\ell)
\end{array}\right) .
\end{aligned}
$$

Proposition 6.1. Let functions $\rho, I_{\rho}, K, E I, p_{1}, p_{2}, h_{1}, h_{2}$ satisfy (6.3a), (6.3b), (6.9), and (6.13). Then the operator $\mathcal{L}$ is similar to the $4 \times 4$ Dirac-type operator $L:=L_{C, D}(Q)$ with the matrices $B, C, D, Q(\cdot)$ given by (6.12a), (6.17) and (6.16).

Proof. Introduce the following operator

$$
U y=\operatorname{col}\left(E I(x) y_{1}^{\prime}, y_{2}, K(x)\left(y_{3}^{\prime}-y_{1}\right), y_{4}\right), \quad y=\operatorname{col}\left(y_{1}, y_{2}, y_{3}, y_{4}\right), \quad(6.18)
$$

that maps the Hilbert space $\mathfrak{H}$ given by $(6.4)$ into $L^{2}\left([0, \ell] ; \mathbb{C}^{4}\right)$. Since $\frac{d}{d x}$ isometrically maps $\widetilde{H}_{0}^{1}[0, \ell]=\left\{f \in W^{1,2}[0, \ell]: f(0)=0\right\}$ onto $L^{2}[0, \ell]$, it follows from 
conditions (6.3b) that the operator $U$ is bounded with bounded inverse. It is easy to check that for $y=\operatorname{col}\left(y_{1}, y_{2}, y_{3}, y_{4}\right)$

$$
\begin{gathered}
\mathcal{L} U^{-1} y=\frac{1}{i}\left(\begin{array}{c}
y_{2} \\
\frac{1}{I_{\rho}}\left(y_{1}^{\prime}-p_{1} y_{2}+y_{3}\right) \\
y_{4} \\
\frac{1}{\rho}\left(y_{3}^{\prime}-p_{2} y_{4}\right)
\end{array}\right), \\
\tilde{L} y:=U \mathcal{L} U^{-1} y=\frac{1}{i}\left(\begin{array}{c}
\frac{1}{I_{\rho}}\left(y_{1}^{\prime}-p_{1} y_{2}+y_{3}\right) \\
K \cdot\left(y_{4}^{\prime}-y_{2}\right) \\
\frac{1}{\rho}\left(y_{3}^{\prime}-p_{2} y_{4}\right)
\end{array}\right),
\end{gathered}
$$

and

$$
\begin{aligned}
& \operatorname{dom}(\tilde{L})=U \operatorname{dom}(\mathcal{L})=\left\{y=\operatorname{col}\left(y_{1}, y_{2}, y_{3}, y_{4}\right) \in W^{1,1}\left([0, \ell] ; \mathbb{C}^{4}\right):\right. \\
& \tilde{L} y \in L^{2}\left([0, \ell] ; \mathbb{C}^{4}\right) \text {, } \\
& y_{2}(0)=y_{4}(0)=0 \text {, } \\
& y_{1}(\ell)+\alpha_{1} y_{2}(\ell)+\beta_{1} y_{4}(\ell)=0 \text {, } \\
& \left.y_{3}(\ell)+\alpha_{2} y_{4}(\ell)+\beta_{2} y_{2}(\ell)=0 .\right\} \text {. }
\end{aligned}
$$

Thus, the operator $\mathcal{L}$ is similar to the operator $\tilde{L}$,

$$
\widetilde{L} y=-i \widetilde{B}(x) y^{\prime}+\widetilde{Q}(x) y
$$

with the domain $\operatorname{dom}(\widetilde{L})$ given by $(6.20)$, and the matrix functions $\widetilde{B}(\cdot), \widetilde{Q}(\cdot)$, given by

$$
\widetilde{B}(x):=\left(\begin{array}{cccc}
0 & E I(x) & 0 & 0 \\
\frac{1}{I_{\rho}(x)} & 0 & 0 & 0 \\
0 & 0 & 0 & K(x) \\
0 & 0 & \frac{1}{\rho(x)} & 0
\end{array}\right),
$$


and

$$
\widetilde{Q}(x):=i\left(\begin{array}{cccc}
0 & 0 & 0 & 0 \\
0 & \frac{p_{1}(x)}{I_{\rho}(x)} & -\frac{1}{I_{\rho}(x)} & 0 \\
0 & K(x) & 0 & 0 \\
0 & 0 & 0 & \frac{p_{2}(x)}{\rho(x)}
\end{array}\right) .
$$

Note, that $\widetilde{Q} \in L^{1}\left([0, \ell] ; \mathbb{C}^{4 \times 4}\right)$ in view of conditions (6.3a)-(6.3b). Next we diagonalize the matrix $\widetilde{B}(\cdot)$. Namely, setting

$$
\tilde{U}(x):=\left(\begin{array}{cccc}
-h_{1}(x) & h_{1}(x) & 0 & 0 \\
1 & 1 & 0 & 0 \\
0 & 0 & -h_{2}(x) & h_{2}(x) \\
0 & 0 & 1 & 1
\end{array}\right),
$$

and noting that

$$
\tilde{U}^{-1}(x)=\frac{1}{2}\left(\begin{array}{cccc}
-\frac{1}{h_{1}(x)} & 1 & 0 & 0 \\
\frac{1}{h_{1}(x)} & 1 & 0 & 0 \\
0 & 0 & -\frac{1}{h_{2}(x)} & 1 \\
0 & 0 & \frac{1}{h_{2}(x)} & 1
\end{array}\right),
$$

we easily get after straightforward calculations

$$
\begin{aligned}
\tilde{U}^{-1}(x) \tilde{B}(x) \tilde{U}(x) & =\operatorname{diag}\left(-\sqrt{\frac{E I(x)}{I_{\rho}(x)}}, \sqrt{\frac{E I(x)}{I_{\rho}(x)}},-\sqrt{\frac{K(x)}{\rho(x)}}, \sqrt{\frac{K(x)}{\rho(x)}}\right) \\
& =\frac{1}{\gamma(x)} B^{-1},
\end{aligned}
$$

Here we have used definition (6.12c) of $h_{1}, h_{2}$, and definitions (6.10) and (6.11) of $b_{1}, b_{2}$, and $\gamma(x)$, respectively. Further, note that $\tilde{U}(\cdot) \in W^{1,1}\left([0, \ell] ; \mathbb{C}^{4 \times 4}\right)$ and $\hat{Q} \in L^{1}\left([0, \ell] ; \mathbb{C}^{4 \times 4}\right)$ in view of $(6.3 \mathrm{a}),(6.3 \mathrm{~b})$ and $(6.13)$, where $\hat{Q}(\cdot)$ is given by (6.14) and (6.12b). Hence, it is easily seen that

$$
\tilde{U}^{-1}(x) \widetilde{Q}(x) \tilde{U}(x)-i \widetilde{U}^{-1}(x) \widetilde{B}(x) \tilde{U}^{\prime}(x)=\hat{Q}(x), \quad x \in[0, \ell] .
$$

Introducing the operator $\tilde{U}: y \rightarrow \tilde{U}(x) y$ in $L^{2}\left([0, \ell] ; \mathbb{C}^{4}\right)$ and taking into account (6.22) and (6.23) we obtain that for any $y \in W^{1,1}\left([0, \ell] ; \mathbb{C}^{4}\right)$ and satisfying $\tilde{U} y \in \operatorname{dom}(\tilde{L})$

$$
\widehat{L} y:=\tilde{U}^{-1} \tilde{L} \tilde{U} y=-i \gamma(x)^{-1} B^{-1} y^{\prime}+\widehat{Q}(x) y .
$$


Next, taking into account formulas (6.17) and (6.21) for matrices $C, D$, and $\widetilde{U}(\cdot)$, respectively, we derive

$$
\operatorname{dom}(\widehat{L})=\left\{y \in W^{1,1}\left([0, \ell] ; \mathbb{C}^{4}\right): \hat{L} y \in L^{2}\left([0, \ell] ; \mathbb{C}^{4}\right), C y(0)+D y(\ell)=0\right\} .
$$

Finally, we apply similarity transformation $S$ that realizes the change of variable $x=x(t)$,

$$
S: L^{2}\left([0, \ell] ; \mathbb{C}^{4}\right) \rightarrow L^{2}\left([0,1] ; \mathbb{C}^{4}\right), \quad(S f)(t)=f(x(t)), \quad t \in[0,1] .
$$

Since both $t(\cdot)$ and $x(\cdot)$ are strictly increasing and continuously differentiable, the following implications hold

$$
\begin{aligned}
& f(\cdot) \in W^{1,1}\left([0, \ell] ; \mathbb{C}^{4}\right) \Longrightarrow f(x(\cdot)) \in W^{1,1}\left([0,1] ; \mathbb{C}^{4}\right), \\
& g(\cdot) \in W^{1,1}\left([0,1] ; \mathbb{C}^{4}\right) \Longrightarrow g(t(\cdot)) \in W^{1,1}\left([0, \ell] ; \mathbb{C}^{4}\right) .
\end{aligned}
$$

Hence (6.25) and (1.5) implies $\operatorname{dom}(L)=S \operatorname{dom}(\widehat{L})$. Next, it follows from (6.15) that $t^{\prime}(x)=\gamma(x), x \in[0, \ell]$. Hence for $f \in \operatorname{dom}(L)$ and $x \in[0, \ell]$ one has

$$
\begin{aligned}
\left(\hat{L} S^{-1} f\right)(x) & =-i \gamma(x)^{-1} B^{-1} \frac{d}{d x}[f(t(x))]+\hat{Q}(x) f(t(x)) \\
& =-i B^{-1} f^{\prime}(t(x))+\hat{Q}(x) f(t(x)),
\end{aligned}
$$

which directly implies that $L=S \widehat{L} S^{-1}$. Combining this identity with (6.19) and (6.24) one concludes that $\mathcal{L}$ is similar to $L=L_{C, D}(Q)$.

Remark 6.2. Proposition 6.1 remains valid if we replace (6.3a) by the weaker assumption $\rho, I_{\rho}, K, E I \in L^{\infty}[0, \ell]$ and assume in addition that the inverse function $x(\cdot)=t^{-1}(\cdot)$ is absolutely continuous. Otherwise implication (6.26) fails, since in general the inverse function of absolutely continuous function is not necessarily absolutely continuous. For instance, the function

$$
h(x):=x+C(x), \quad x \in[0,1],
$$

where $C(\cdot)$ is the Cantor function, strictly increases and is not absolutely continuous. At the same time, the inverse function is absolutely continuous.

Applying [37, Corollary 3.2] and Theorem 5.6 to the operator $L$ we obtain the following result. 
Theorem 6.3. Let conditions (6.3a), (6.3b), (6.9), (6.13) be satisfied and let also

$$
\left(\alpha_{1}+h_{1}(\ell)\right)\left(\alpha_{2}+h_{2}(\ell)\right) \neq \beta_{1} \beta_{2}
$$

and

$$
\left(\alpha_{1}-h_{1}(\ell)\right)\left(\alpha_{2}-h_{2}(\ell)\right) \neq \beta_{1} \beta_{2}
$$

(i) Then the system of root functions of $\mathcal{L}$ is complete and minimal in $\mathfrak{H}$.

(ii) Assume in addition that

$$
p_{1}, p_{2} \in L^{\infty}[0, \ell], \quad h_{1}, h_{2} \in \operatorname{Lip}_{1}[0, \ell], \quad \beta_{1}=\beta_{2}=0
$$

Then the system of root functions of the operator $\mathcal{L}$ forms a Riesz basis with parentheses in $\mathfrak{H}$.

Proof. (i) Consider the operator $L_{C, D}(Q)$ defined in Proposition 6.1. Combining expressions (6.12a) and (6.17) for the matrices $B, C, D$ with definition of $T_{A}(C, D)$ yields

$$
\begin{aligned}
\operatorname{det} T_{B}(C, D) & =\operatorname{det}\left(\begin{array}{cccc}
1 & 0 & 0 & 0 \\
0 & \alpha_{1}+h_{1}(\ell) & 0 & \beta_{1} \\
0 & 0 & 1 & 0 \\
0 & \beta_{2} & 0 & \alpha_{2}+h_{2}(\ell)
\end{array}\right) \\
& =\left(\alpha_{1}+h_{1}(\ell)\right)\left(\alpha_{2}+h_{2}(\ell)\right)-\beta_{1} \beta_{2} .
\end{aligned}
$$

Similarly one gets $\operatorname{det} T_{-B}(C, D)=\left(\alpha_{1}-h_{1}(\ell)\right)\left(\alpha_{2}-h_{2}(\ell)\right)-\beta_{1} \beta_{2}$. Conditions (6.29) implies $\operatorname{det} T_{B}(C, D) \neq 0$ and $\operatorname{det} T_{-B}(C, D) \neq 0$. Therefore, by [37, Corollary 3.2], the system of root functions of the operator $L_{C, D}(Q)$ is complete and minimal in $L^{2}\left([0,1] ; \mathbb{C}^{4}\right)$. Since, by Proposition $6.1, \mathcal{L}$ is similar to the operator $L_{C, D}(Q)$, the system of root functions of the operator $\mathcal{L}$ is complete and minimal in $\mathfrak{H}$.

(ii) Again consider the operator $L_{C, D}(Q)$ defined in Proposition 6.1. Since $\beta_{1}=\beta_{2}=0$ and (6.29) is fulfilled, then according to (6.12a) and (6.17) the matrices $B, C, D$ have the block structure described in (5.10)-(5.11) with $r=2$. Moreover, (6.30) implies $Q \in L^{\infty}\left([0,1] ; \mathbb{C}^{4 \times 4}\right)$. Therefore, combining Theorem 5.6 with Proposition 6.1 yields the statement. 
Applying Corollary 4.9 we can improve Theorem 6.3(i) assuming that $\hat{Q}(\cdot)$ is continuous at the endpoints $0, \ell$. For simplicity we assume that $\beta_{1}=\beta_{2}=0$.

Theorem 6.4. Let the functions $\rho, I_{\rho}, K, E I, p_{1}, p_{2}, h_{1}, h_{2}$ satisfy (6.3a), (6.3b), (6.9), and (6.13). Let also the functions $p_{1}, p_{2}, h_{1}^{\prime}, h_{2}^{\prime}$ be continuous at the endpoints 0 and $\ell$. Assume in addition that $\beta_{1}=\beta_{2}=0$ and the following assumptions are fulfilled:

(i) $\left|\alpha_{1}-h_{1}(\ell)\right|+\left|\alpha_{2}-h_{2}(\ell)\right| \neq 0$ and $\left|\alpha_{1}+h_{1}(\ell)\right|+\left|\alpha_{2}+h_{2}(\ell)\right| \neq 0$;

(ii) for each $j \in\{1,2\}$ one of the following conditions is satisfied:

(a) $\alpha_{j}^{2} \neq h_{j}^{2}(\ell)$

(b) $\alpha_{j}=h_{j}(\ell)$ and $h_{j}^{\prime}(\ell) \neq-p_{j}(\ell)$;

(c) $\alpha_{j}=-h_{j}(\ell)$ and $h_{j}^{\prime}(\ell) \neq p_{j}(\ell)$.

Then the system of root functions of $\mathcal{L}$ is complete and minimal in $\mathfrak{H}$.

Proof. Consider the operator $L_{C, D}(Q)$ defined in Proposition 6.1. Since $\rho, I_{\rho} \in$ $C[0, \ell]$ and $p_{1}, p_{2}, h_{1}^{\prime}, h_{2}^{\prime}$ are continuous at the endpoints 0 and $\ell$, it follows from $(6.12 \mathrm{~b})-(6.16)$ that the matrix function $Q(\cdot)$ is continuous at the endpoints 0 and 1. Since $\beta_{1}=\beta_{2}=0$, the block matrix representations (6.12a) and (6.17) of the matrices $B, C, D$, allow to apply Corollary 4.9 and Lemma 4.10. Let us verify conditions (4.10)-(4.13) of Lemma 4.10. First, comparing (4.6) with (6.17) yields

$$
\begin{array}{ll}
d_{1}=\alpha_{1}-h_{1}(\ell), & d_{2}=\alpha_{1}+h_{1}(\ell), \\
d_{3}=\alpha_{2}-h_{2}(\ell), & d_{4}=\alpha_{2}+h_{2}(\ell) .
\end{array}
$$

Therefore, condition (4.10) is always satisfied, since $h_{j}(\ell) \neq 0, j \in\{1,2\}$, while condition (4.11) is equivalent to the condition (i) of the theorem. Further, it follows from (6.14) and (6.16) that

$$
\begin{array}{ll}
q_{12}(1)=\frac{p_{1}(\ell)-h_{1}^{\prime}(\ell)}{-2 i I_{\rho}(\ell)}, & q_{21}(1)=\frac{p_{1}(\ell)+h_{1}^{\prime}(\ell)}{-2 i I_{\rho}(\ell)}, \\
q_{34}(1)=\frac{p_{2}(\ell)-h_{2}^{\prime}(\ell)}{-2 i \rho(\ell)}, & q_{43}(1)=\frac{p_{2}(\ell)+h_{2}^{\prime}(\ell)}{-2 i \rho(\ell)} .
\end{array}
$$

Hence, conditions (4.12) and (4.13) are equivalent to the conditions (a)-(c) of the theorem for $j=1$ and $j=2$, respectively. Therefore, by Lemma 4.10, condition (4.7) is satisfied and, by Corollary 4.9, the system of root functions of the operator $L_{C, D}(Q)$ is complete and minimal in $L^{2}\left([0,1] ; \mathbb{C}^{4}\right)$. Therefore, Proposition 6.1 completes the proof. 
Remark 6.5. The main results remain also valid if the function $v(\cdot)$ given by (6.9) satisfies $v(x) \neq 1$ for $x \in[0, \ell]$.

Remark 6.6. (i) In connection with Theorem 6.3 we mention the paper [54] where the operator $\mathcal{L}$ was investigated under the following assumptions on the parameters of the model:

$$
E I, K \in W^{3,2}[0, \ell], \quad \rho, I_{\rho} \in W^{4,2}[0, \ell], \quad p_{1}=p_{2}=0, \quad \beta_{1}=\beta_{2}=0,
$$

but without the algebraic assumption (6.9). The completeness of the root functions was stated in [54] under the condition (6.29) and the additional assumption

$$
I_{\rho}(x) K(x) \neq \rho(x) E I(x), \quad x \in[0, \ell],
$$

which in our notations means that $v(x) \neq 1, x \in[0, \ell]$. Unfortunately, the proof of the completeness in [54] fails because of the incorrect application of the Keldysh theorem. Namely, the representation $\mathcal{L}^{-1}=\mathcal{L}_{00}^{-1}\left(I_{\mathfrak{H}}+T\right)$ used in [54], where $T$ is of finite rank bounded operator and $\mathcal{L}_{00}=\mathcal{L}_{00}^{*}$, fails since it leads to the inclusion $\operatorname{dom}(\mathcal{L}) \subset \operatorname{dom}\left(\mathcal{L}_{00}\right)$, which holds if only if $\mathcal{L}=\mathcal{L}_{00}$.

Moreover, under conditions (6.32), (6.33) and (6.29) the Riesz basis property for the system of root functions of $\mathcal{L}$ was stated in [54]. The proof is based on the claim that under the above restrictions the eigenvalues of $\mathcal{L}$ are asymptotically simple and separated. However, it is not the case. For instance, if $K \equiv E I \equiv \rho \equiv$ $1, I_{\rho} \equiv 4, \alpha_{1}=5 / 2$ and $\alpha_{2}=13 / 12$, then according to [54, Theorem 4.2] the sequence of the eigenvalues of $\mathcal{L}$ splits into two families

$\lambda_{n}^{(1)}=\frac{\pi n}{2}+\frac{i}{2} \ln 3+O\left(n^{-1}\right) \quad$ and $\quad \lambda_{n}^{(2)}=\pi n+\frac{i}{2} \ln 3+O\left(n^{-1}\right), \quad n \in \mathbb{Z} \backslash\{0\}$.

Clearly, in this case the sequence of the eigenvalues of $\mathcal{L}$ is not asymptotically simple and separated. Note, however, that according to Theorem 6.3(ii) the system of root functions of the operator $\mathcal{L}$ always forms a Riesz basis with parentheses under the restrictions (6.3a), (6.3b), (6.9), (6.13), (6.29) and (6.30).

(ii) In connection with Theorem 6.3 we also mention the paper [61]. In this paper the operator $\mathcal{L}$ was investigated under the following stronger assumptions on the parameters of the model:

$$
\begin{array}{rr}
E I, K, \rho, I_{\rho} \text { are constant, } & p_{1}=p_{2}=0, \\
\alpha_{1}, \alpha_{2}, \beta_{1}, \beta_{2} \geqslant 0, & 4 \alpha_{1} \alpha_{2} \geqslant\left(\beta_{1}+\beta_{2}\right)^{2} .
\end{array}
$$


The last condition in (6.34) ensures the dissipativity of the operator $\mathcal{L}$. The completeness of the system of root functions of the operator $\mathcal{L}$ was proved in [61] under the restrictions (6.34) and (6.29). So, our Theorem 6.3(i) generalizes this result to a broader class of boundary conditions and improves it in the dissipative case. Note also that under additional assumptions, guarantying that the eigenvalues of $\mathcal{L}$ are asymptotically simple and separated, it was proved in [61] that the root functions of $\mathcal{L}$ contains the Riesz basis. Moreover, this fact was applied to show the exponential stability of the problem (6.1a)-(6.2c).

Acknowledgments. We are indebted to D. Yakubovich for the reformulation of the condition (a) of Theorem 4.1 mentioned in Remark 4.2. We are also indebted to the anonymous referee for useful remarks helping us to improve the exposition.

\section{References}

[1] A. V. Agibalova, M. M. Malamud, and L. L. Oridoroga, On the completeness of general boundary value problems for $2 \times 2$ first-order systems of ordinary differential equations. Methods Funct. Anal. and Topology 18 (2012), no. 1, 4-18. MR 2953328 Zbl 1249.34249

[2] A. G. Baskakov, A. V. Derbushev, and A. O. Shcherbakov, The method of similar operators in the spectral analysis of non-self-adjoint Dirac operators with non-smooth potentials. Izv. Ross. Akad. Nauk Ser. Mat. 75 (2011), no. 3, 3-28. In Russian. English trans. Izv. Math. 75 (2011), no. 3, 445-469. MR 2847780 Zbl 1219.47024

[3] G. D. Birkhoff, On the asymptotic character of the solutions of certain linear differential equations containing a parameter. Trans. Amer. Math. Soc. 9 (1908), no. 2, 219-231. MR 1500810

[4] G. D. Birkhoff, Boundary value and expansion problems of ordinary linear differential equations. Trans. Amer. Math. Soc. 9 (1908), no. 4, 373-395. MR 1500818 JFM 39.0386.02

[5] G. D. Birkhoff and R. E. Langer, The boundary problems and developments associated with a system of ordinary differential equations of the first order. Proc. Amer. Acad. Arts Sci. 58 (1923), no. 2, 49-128. JFM 49.0723.01

[6] P. Djakov and B. Mityagin, Bari-sMarkus property for Riesz projections of 1D periodic Dirac operators. Math. Nachr. 283 (2010), no. 3, 443-462. MR 2643021 Zbl 1198.34188

[7] P. Djakov and B. Mityagin, Unconditional convergence of spectral decompositions of 1D Dirac operators with regular boundary conditions. Indiana Univ. Math. J. 61 (2012), no. 1, 359-398. MR 3029402 Zbl 1297.47047

[8] P. Djakov and B. Mityagin, Convergence of spectral decompositions of Hill operators with trigonometric polynomial potentials. Math. Ann. 351 (2011), no. 3, 509-540. MR $2854104 \mathrm{Zbl} 05988211$ 
[9] P. Djakov and B. Mityagin, 1D Dirac operators with special periodic potentials. Bull. Pol. Acad. Sci. Math. 60 (2012), no. 1, 59-75. MR 2901387 Zbl 1256.47029

[10] P. Djakov and B. Mityagin, Equiconvergence of spectral decompositions of 1D Dirac operators with regular boundary conditions. J. Approx. Theory 164 (2012), no. 7, 879-927. MR 2922621 Zbl 1258.34174

[11] P. Djakov and B. Mityagin, Criteria for existence of Riesz bases consisting of root functions of Hill and 1D Dirac operators. J. Funct. Anal. 263 (2012), no. 8, 2300-2332. MR 2964684 Zbl 1263.34121

[12] P. Djakov and B. Mityagin, Riesz bases consisting of root functions of 1D Dirac operators. Proc. Amer. Math. Soc. 141 (2013), no. 4, 1361-1375. MR 3008883 Zbl 1297.47046

[13] N. Dunford, A survey of the theory of spectral operators. Bull. Amer. Math. Soc. 64 (1958), 217-274. MR 0104865 Zbl 0088.32102

[14] N. Dunford and J. Schwartz, Linear operators. Part III. Spectral operators. With the assistance of W. G. Bade and R. G. Bartle. Pure and Applied Mathematics, Vol. VII. Interscience Publishers (John Wiley \& Sons), New York etc., 1971. MR 0412888 Zbl 0243.47001

[15] F. Gesztesy and V. Tkachenko, A criterion for Hill operators to be spectral operators of scalar type. J. Anal. Math. 107 (2009), 287-353. MR 2496408 Zbl 1193.47037

[16] F. Gesztesy and V. Tkachenko, A Schauder and Riesz basis criterion for non-selfadjoint Schrödinger operators with periodic and antiperiodic boundary conditions. J. Differential Equations 253 (2012), no. 2, 400-437. MR 2921200 Zbl 1251.34100

[17] Yu. P. Ginzburg, The almost invariant spectral propeties of contractions and the multiplicative properties of analytic operator-functions. Funkcional. Anal. i Priložen. 5 (1971) no. 3, 32-41. In Russian. English transl., Funct. Anal. Appl. 5 (1971), no. 3, 197-205. MR 0283604

[18] I. C. Gohberg and M. G. Krein, Introduction to the theory of linear non-selfadjoint operators in Hilbert space. (Введение в теорию линейных несамосопряженных операторов в гильбертовом пространстве.) Nauka, Moskow, 1965. In Russian. English trasl., Transl. Math. Monographs 18. American Mathematical Society, Providence, R.I. 1969. MR 0220070 Zbl 0138.07803

[19] G. M. Gubreev, On the spectral decomposition of finite-dimensional perturbations of dissipative Volterra operators. Tr. Mosk. Mat. Obs. 64 (2003), 90-140. In Russian. English transl., Trans. Moscow Math. Soc. 2003 (2003), 79-126. MR 2030187 Zbl 1083.47505

[20] S. Hassi and L. Oridoroga, Theorem of completeness for a Dirac-type operator with generalized $\lambda$-depending boundary conditions. Integral Equations Operator Theory 64 (2009), no. 3, 357-379. MR 2521241 Zbl 1195.34131

[21] M. V. Keldyš, On the characteristic values and characteristic functions of certain classes of non-self-adjoint equations. Doklady Akad. Nauk SSSR (N.S.) 77, (1951). 11-14. In Russian. MR 0041353 Zbl 0045.39402 
[22] G. M. Kesel'man, On the unconditional convergence of eigenfunction expansions of certain differential operators. Izv. Vyš̌. Učebn. Zaved. Matematika 1964 (1964), no. 2(39), 82-93. MR 0166630 Zbl 0135.29701

[23] A. P. Khromov, Finite dimensional perturbations of Volterra operators. Sovrem. Mat. Fundam. Napravl. 10 (2004), 3-163. In Russian. English transl., J. Math. Sci. (N. Y.) 138 (2006), no. 5, 5893-6066. MR 2120867 Zbl 1090.47007

[24] J. U. Kim and Y. Renardy, Boundary control of the Timoshenko beam. SIAM J. Control Optim. 25 (1987), no. 6, 1417-1429. MR 0912448 Zbl 0632.93057

[25] A. G. Kostyuchenko and A. A. Shkalikov, Summability of expansions in eigenfunctions of differential operators and of convolution operators. Funktsional. Anal. $i$ Prilozhen. 12 (1978), no. 4, 24-40. In Russian. English transl., Functional Anal. Appl. 12 (1978), no. 4, 262-276 (1979). MR 0515627 Zbl 0394.34018

[26] B. Ya. Levin, Lectures on entire functions. In collaboration with and with a preface by Yu. Lyubarskii, M. Sodin, and V. Tkachenko. Translated from the Russian manuscript by Yu. Tkachenko. Translations of Mathematical Monographs, 150. American Mathematical Society, Providence, RI, 1996. MR 1400006 Zbl 0856.30001

[27] B. M. Levitan and I. S. Sargsyan, Sturm-Liouville and Dirac operators. Nauka, Moscow, 1988. In Russian. English transl. by O. Efimov, Mathematics and its Applications (Soviet Series), 59. Kluwer Academic Publishers Group, Dordrecht, 1991. MR 0958344 MR 1136037 (transl.) Zbl 0657.34002 Zbl 0704.34002 (transl.)

[28] A. A. Lunyov and M. M. Malamud, On the completeness of the root vectors for first order systems. Dokl. Akad. Nauk, Ross. Akad. Nauk 453 (2013), no. 3, 256-261. In Rusian. English transl., Dokl. Math. 88 (2013), no. 3, 678-683. MR 3156298 Zbl 06336234

[29] A. A. Lunyov and M. M. Malamud, On spectral synthesis for dissipative Dirac type operators. Integral Equations Operator Theory 80 (2014), no. 1, 79-106. MR 3248475 Zbl 06340738

[30] A. A. Lunyov and M. M. Malamud, On the Riesz basis property of the root vector system for Dirac-type $2 \times 2$ systems. Dokl. Akad. Nauk, Ross. Akad. Nauk 458 (2014), no. 3, 255-260. In Russian. English transl., Dokl. Math. 90 (2014), no. 2, 556-561. Zbl 06397160

[31] A. A. Lunyov and M. M. Malamud, On the completeness and Riesz basis property of root subspaces of boundary value problems for first order systems. Preprint 2014. arXiv:1401.2574 [math.SP]

[32] Yu. I. Lyubarskiǔ and V. A. Tkachenko, System $\left\{e^{\alpha n x} \sin n x\right\}$. Funktsional. Anal. $i$ Prilozhen. 18 (1984), no. 2, 69-70. English transl., Functional Anal. Appl. 18 (1984), no. 2, 144-146. MR 0745708 Zbl 0545.42021 Zbl 0601.42031 (transl.)

[33] A. S. Makin, On summability of spectral expansions corresponding to the SturmLiouville operator. Int. J. Math. Math. Sci. 2012 (2012), Article Id. 843562, 13 pp. MR 2974689 Zbl 06093869 
[34] M. M. Malamud, Questions of uniqueness in inverse problems for systems of differential equations on a finite interval. Tr. Mosk. Mat. Obs. 60 (1999), 199-258. In Russian. English transl., Trans. Moscow Math. Soc. 1999 (1999), 173-224. MR 1702668 Zbl 0939.34012

[35] M. M. Malamud, On the completeness of a system of root vectors of the Sturm-Liouville operator with general boundary conditions. Funktsional. Anal. $i$ Prilozhen. 42 (2008), no. 3, 45-52, 96. In Russian. English transl., Funct. Anal. Appl. 42 (2008), no. 3, 198-204. MR 2454475 Zbl 1167.34393

[36] M. M. Malamud and L. L. Oridoroga, Completeness theorems for systems of differential equations. Funktsional. Anal. i Prilozhen. 34 (2000), no. 4, 88-90. In Russian. English transl., Funct. Anal. Appl. 34 (2000), no. 4, 308-310. MR 1819650 Zbl 0979.34058

[37] M. M. Malamud and L. L. Oridoroga, On the completeness of root subspaces of boundary value problems for first order systems of ordinary differential equations. J. Funct. Anal. 263 (2012), no. 7, 1939-1980. MR 1256.34075 Zbl 2956931

[38] V. A. Marchenko, Sturm-Liouville operators and applications. Translated from the Russian by A. Iacob. Operator Theory: Advances and Applications, 22. Birkhäuser Verlag, Basel, 1986. MR 0897106 Zbl 0592.34011

[39] A. S. Markus, An introduction to the spectral theory of polynomial operator pencils. Shtiintsa, Chisinau, 1986. In Russian.

[40] A. S. Markus and V. I. Matsaev, Comparison theorems for spectra of linear operators and spectral asymptotics. Trudy Moskov. Mat. Obshch. 45 (1982), 133-181. In Russian. English transl., Trans. Mosc. Math. Soc. 1984 (1984), no. 1, 139-187. MR 0704630 Zbl 0532.47012 Zbl 0557.47009 (transl.)

[41] V. P. Mihaŭlov, On Riesz bases in $L^{2}(0,1)$. Dokl. Akad. Nauk SSSR 144 (1962), 981-984. In Russian. MR 0149334

[42] A. Minkin, Spectrality of ordinary differential operators. Preprint 2005. arXiv:math/0409181v2 [math.SP]

[43] A. Minkin, Resolvent growth and Birkhoff-regularity. J. Math. Anal. Appl. 323 (2006), no. 1, 387-402. MR 2262213 Zbl 1114.34064

[44] Ya. V. Mykytyuk, D. V. Puyda, Bari-Markus property for Dirac operators. Mat. Stud. 40 (2013), no. 2, 165-171. MR 3185251 Zbl 06387479

[45] M. A. Naimark, Linear differential operators. I. I: Elementary theory of linear differential operators. English transl. by E. R. Dawson edited by W. N. Everitt. With additional material by the author. Frederick Ungar, New York, 1967. MR 0216050 Zbl 0219.34001

[46] S. P. Novikov, S. V. Manakov, L. P. Pitaevskij, and V. E. Zakharov, Theory of solitons. The inverse scattering method. (Теория солитонов. Метод обратной задачи.) Nauka, Moscow, 1980. In Russian. English transl., Contemporary Soviet Mathematics. Consultants Bureau [Plenum], New York, 1984. MR 0573607 MR 0779467 (transl.) Zbl 0598.35003 Zbl 0598.35002 (transl.) 
[47] V. S. Rykhlov, Completeness of eigenfunctions of one class of pencils of differential operators with constant coefficients. Izv. Vyssh. Uchebn. Zaved. Mat. 2009 (2009), no. 6, 42-53. In Russian. Englis transl., Russian Math. (Iz. VUZ) 53 (2009), no. 6, 33-43. MR 2583998 Zbl 1190.34109

[48] A. M. Savchuk and A. A. Shkalikov, The Dirac operator with complex-valued summable potential. Math Notes 96 (2014), no. 5-6, 777-810.

[49] A. A. Shkalikov, The completeness of the eigen- and associated functions of an ordinary differential operator with nonregular splitting boundary conditions. Funct. Anal. Appl. 10 (1976), no. 4 305-316.

[50] A. A. Shkalikov, The basis problem of the eigenfunctions of ordinary differential operators with integral boundary conditions. Vestnik Moskov. Univ. Ser. I Mat. Mekh. 1982 (1982), no. 6, 12-21, 120. In Russian. English transl., Moscow Univ. Math. Bull. 37 (1982), no. 6, 10-20. MR 0685257 Zbl 0565.34020

[51] A. A. Shkalikov, Boundary problems for ordinary differential equations with parameter in the boundary conditions Trudy Sem. Petrovsk. 9 (1983), 190-229. In Russian. English transl., J. Soviet Math. 33 (1986), no. 6, 1311-1342. MR 0731903 Zbl 0553.34014 Zbl 0609.34019 (transl.)

[52] A. A. Shkalikov, On the basis property of root vectors of a perturbed self-adjoint operator. Tr. Mat. Inst. Steklova 269 (2010), Teoriya Funktsii i Differentsialnye Uravneniya, 290-303. In Russian. English transl., Proc. Steklov Inst. Math. 269 (2010), no. 1, 284-298. MR 2729992 Zbl 1200.47021

[53] A. A. Shkalikov and O. A. Veliev, On the Riesz basis property of the eigen- and associated functions of periodic and antiperiodic Sturm-Liouville problems. Mat. Zametki 85 (2009), no. 5, 671-686. In Russian. English transl., Math. Notes 85 (2009), no. 5-6, 647-660. MR 2572858 Zbl 1190.34111

[54] M. A. Shubov, Asymptotic and spectral analysis of the spatially nonhomogeneous Timoshenko beam model. Math. Nachr. 241 (2002), 125-162. MR 1912383 Zbl 1037.47032

[55] J. D. Tamarkin, On some general problems of the theory of ordinary linear differential operators and the expansion of arbitrary functions into series. Petrograd 1917.

[56] J. D. Tamarkin, Some general problems of the theory of linear differential equations and expansions of an arbitrary functions in series of fundamental functions. Math. Z. 27 (1928), no. 1, 1-54. MR 1544895

[57] S. Timoshenko, Vibration problems in engineering. Constable and Co., London, 1937. JFM 63.1305.03

[58] I. Trooshin and M. Yamamoto, Spectral properties and an inverse eigenvalue problem for nonsymmetric systems of ordinary differential operators. J. Inverse Ill-Posed Probl. 10 (2002), no. 6, 643-658. MR 1967444 Zbl 1038.34012

[59] Y. Wu and X. Xue, Decay rate estimates for the quasi-linear Timoshenko system with nonlinear control and damping terms. J. Math. Phys. 52 (2011), no. 9, Article Id. 093502, 18 pp. MR 2867820 Zbl 1272.74391 
[60] G. Q. Xu, Z. J. Han, and S. P. Yung, Riesz basis property of serially connected Timoshenko beams. Internat. J. Control 80 (2007), no. 3, 470-485. MR 2294162 Zbl 1120.93026

[61] G. Q. Xu and S. P. Yung, Exponential decay rate for a Timoshenko beam with boundary damping. J. Optim. Theory Appl. 123 (2004), no. 3, 669-693. MR 2102537 Zbl 1068.35172

Received December 23, 2013; revised August 24, 2013

Anton A. Lunyov, Institute of Applied Mathematics and Mechanics NAS of Ukraine, Roza Luzemburg str. 74, 83114 Donetsk, Ukraine

e-mail: A.A.Lunyov@gmail.com

Mark M. Malamud,

Institute of Applied Mathematics and Mechanics NAS of Ukraine,

Roza Luzemburg str. 74, 83114 Donetsk, Ukraine

e-mail:mmm@telenet.dn.ua 